\title{
The P-Hub maximal covering problem and extensions for gradual decay functions ${ }^{2}$
}

\author{
Meltem Peker, Bahar Y. Kara* \\ Department of Industrial Engineering, Bilkent University, 06800 Ankara, Turkey
}

\section{A R T I C L E I N F O}

Article history:

Received 23 December 2013

Accepted 20 January 2015

This manuscript was processed by Associate

Editor Salazar-Gonzalez.

Available online 2 February 2015

Keywords:

Hub location problem

p-hub maximal covering problem

Partial coverage

\begin{abstract}
A B S T R A C T
The $p$-hub maximal covering problem aims to find the best locations for hubs so as to maximize demands within a coverage distance with a predetermined number of hubs. Classically, the problem is defined in the framework of binary coverage only; an origin-destination pair is covered if the cost (time, etc.) is lower than the critical value, and not covered at all if the cost is greater than the critical value. In this paper, we extend the definition of coverage, introducing "partial coverage", which changes with distance. We present new and efficient mixed-integer programming models that are also valid for partial coverage for single and multiple allocations. We present and discuss the computational results with different data sets.
\end{abstract}

(c) 2015 Elsevier Ltd. All rights reserved.

\section{Introduction}

Hubs are special facilities that serve as switching, transshipment and sorting centers in many-to-many distribution systems. The main advantage of hubs is their efficiency due to the economies of scale achieved by consolidating flows at the hubs. In "classical" hub location problems, the cost between any two hubs is reduced by a discount factor, $\alpha$, whereas variable discount factors changing with the flow on the links are also used in the literature. Consolidating flows also enables us to use fewer links in the network. The hub location problem includes selecting the location of hub facilities and assigning demand nodes to these hubs to route the flow for each origin-destination $(O-D)$ pair. For flow routing, two types of assignment structures are defined. In single allocation, each node is served by a single hub and all the incoming/outgoing flows of each node are routed through that hub. In multiple allocation, flows can be sent and received through more than one hub.

The hub location problem is first proposed by O'Kelly [25], then garners great interest with the research of Campbell [6]. Campbell [6] classifies hub location problems into four categories with respect to their objectives: $p$-hub median, the uncapacitated hub location, $p$-hub center and hub covering problems. The aim of the $p$-hub median problem is to minimize total transportation cost, and the aim of the uncapacitated hub location problem is to

\footnotetext{
This manuscript was processed by Associate Editor Salazar-Gonzalez.

* Corresponding author. Tel.: +90312 2903156.

E-mail addresses: meltem.peker@bilkent.edu.tr (M. Peker), bkara@bilkent.edu.tr (B.Y. Kara).
}

minimize total transportation cost plus the fixed cost for locating hub facilities. The $p$-hub center problem minimizes maximum distance or service time. The hub covering problem minimizes the number of hubs while satisfying a service requirement for all $O-D$ pairs; i.e. the distance between any $O-D$ pair through located hubs should be shorter than a predetermined distance. Whereas the first two problems focus on economic objectives, usually the $p$ hub center and hub covering problems focus on service level.

Most hub location studies consider complete networks, but some do consider incomplete networks $[13,23]$. Alternative approaches are presented to model economies of scale; flow dependent discount factor [27] or hub arc models [9]. The studies also vary in their solution techniques. Calik et al. [5] propose a tabu-search-based heuristic for the hub covering problem and Chen [10] solves the uncapacitated hub location problem with a hybrid heuristic based on simulated annealing and a tabu search. See Alumur and Kara [2], Campbell and O'Kelly [8] and Kara and Taner [19] for more information about the various hub location problems and solutions.

In this paper, we study the $p$-hub maximal covering problem, which is considered a special type of hub covering problem. The first formulation of the hub covering problem is provided by Campbell [6]. Kara and Tansel [21] provide different linearizations of the original quadratic formulation and propose a new formulation for the single assignment hub covering problem. They also prove that the problem is NP-Hard. Later, Wagner [30] improves the model given in [21] and provides new formulations for the hub covering problem. Ernst et al. [15] show that the formulation in [30] can be further tightened by lifting some of the constraints. They also propose a new formulation for the single allocation version of the problem. 
The $p$-hub maximal covering problem maximizes the demands that are covered with a predetermined number of hubs. The problem for both allocations is posed by Campbell [6]. Later, Hwang and Lee [18] propose a new model for the single assignment version of the problem with $O\left(n^{4}\right)$ variables and constraints. For the multiple assignment version of the problem, Weng et al. [31] develop a new formulation with $O\left(n^{2}\right)$ variables and constraints. The authors also prove the NP-Hardness of the problem. $\mathrm{Qu}$ and Weng [28] use the formulation in [31] in their solution to the problem.

Similar to the hub center and hub covering problems, the p-hub maximal covering problem has a service-oriented objective. Although the problems with economic objectives (i.e. the $p$-hub median problem) are applicable, the solutions can sometimes lead to unsatisfactory results in terms of service level [14]. Therefore, for some sectors better service may be preferable to lower costs. For example, in cargo delivery, firms may choose shorter travel times over lower costs. More-detailed discussions on the $p$-hub median and the $p$-hub maximal covering problems are presented in Section 6.3.

The above-noted papers on the $p$-hub maximal covering problem are only conducted in the framework of binary coverage; any $O-D$ pair is covered if the "cost" (time, etc.) of the path is within the critical value, referred to as $\beta$, and the $O-D$ pair is not covered at all if the cost exceeds the critical value. However, such an assumption for coverage may not be always realistic. The notion of coverage may change drastically, even with an incremental change in the critical value $(\beta)$. For example, i.e. if the cost of a path is $\beta-\varepsilon$, it is considered as "fully covered", but if the cost is $\beta+\varepsilon$, it is considered as "not covered". Therefore, instead of binary (or constant) coverage, "partial coverage", which changes with distance, may sometimes yield more realistic solutions.

Partial coverage has been studied in the context of the covering problem in the general location literature. The maximal covering problem with binary coverage is first defined by Church and ReVelle [11], with binary definitions for "fully covered" and "not covered" nodes. Church and Roberts [12] present the first idea of partial coverage and introduce "partially covered" nodes. They develop a set of new models using step-wise piecewise linear coverage functions. Since binary coverage does not allow one to differentiate the coverage with respect to distance or time; in real life, there may be some cases where the coverage for each zone is different. For example, while the nearest zone's demand is considered fully covered, the farthest zone's demand may only be partially covered. Therefore, to imitate the real life, Berman and Krass [3] formulate the same problem by defining $k$ different zones for coverage and $k$ different radii for each demand node. The coverage function is defined as a nonincreasing step function; the nodes in the first zone are fully covered and beyond the $k$ th zone are not covered at all. Later, this problem is generalized for different types of nonincreasing decay functions by Berman et al. [4]. To solve the problem, Adenso-Diaz and Rodriguez [1] use a tabu search metaheuristic, Galvao and ReVelle [17] use a Lagrangean heuristic for the maximal covering problem with binary coverage. For partial coverage, Karasakal and Karasakal [22] provide a solution procedure based on a Lagrangean relaxation. More information can be found about the extensions of the covering problem in a recent review by Farahani et al. [16].

Similarly, the deficiency of the binary coverage also exists in the $p$-hub maximal covering problem. Instead of binary coverage, partial coverage can be utilized and it may yield higher profit or better customer service level. For instance, in the cargo delivery sector, customers are time sensitive and are looking for fast and punctual delivery services. Therefore, in today's competitive environment, companies try to decrease the time frame within which packages are guaranteed to be delivered. Classically, with binary coverage, zones outside the critical value are not covered and therefore not served. Therefore, the company loses all cargo from these noncovered customers. However, with partial coverage, due to other factors (being a reliable company, etc.), some portion of the noncovered customers may choose to ship their cargo with that company and accept a longer time frame. Hence, the company loses only some of the customers and can make more revenue than with binary coverage. In addition, to attract more customers and encourage them to accept a longer time frame, the carrier may charge less than competitors. For economic reasons, the carrier may still choose not to cover everybody; that is, the carrier may choose to serve only to a threshold value (i.e. serving a small demand for a longer time frame may not profitable for the carrier). Hence, determining the zones that are covered fully or partially and how far to extend partial coverage may depend on economic and competitive issues.

To the best of our knowledge, there is no study on partial coverage in the hub location literature. In this paper, we first relax the definition of binary coverage that has been used in the hub location literature and extend partial coverage to hub location problems. Second, we provide efficient formulations for the $p$-hub maximal covering problem that can be readily applied with partial coverage. We also provide the NP-Hardness proofs of both coverage types on both allocation versions of the hub covering problem.

There are some research where the general assumption on the economies of scale (constant and flow independent) is challenged by showing that there can be more flow on the some spoke links (link between origin/destination and hub) than the flow on the links between hubs [7]. In order to better represent the economies of scale, hub arc type models [9] can be utilized. However in this paper, the main motivation is to provide efficient formulations to the standard $p$-hub maximal covering problems and extend them with the partial coverage notion. Even though this constant economies of scale limits the current work, we aim to develop the idea of partial coverage to the hub location literature. One can extend the notions developed here to flow dependent scale factor or hub arc models.

The rest of the paper is organized as follows: In the next two sections, we provide the new mathematical programming formulations of the $p$-hub maximal covering problem for single and multiple allocations, respectively. In Section 4, we prove that the single allocation version of the problem is NP-Hard even if the hub locations are given. We also present an alternative proof for the multiple allocation version of the problem. We provide the computational results of the new formulations and comparisons with the existing formulations with both binary and partial coverage types for single allocation in Section 5 and for multiple allocation in Section 6. We also discuss the effect of allowing partial coverage to the objective value and hub locations. The paper concludes with Section 7.

\section{Single allocation $\mathbf{p - H u b}$ maximal covering problem (SApHMCP)}

\subsection{Model development of SApHMCP}

Let $N$ be the demand node set, $H$ be the set of potential hubs $(H \subseteq N)$ and the graph be complete and directed. The flow of demand between each $O-D$ pair $i-j$ is denoted by $w_{i j}$. Also, $c_{i j}^{k m}$ represents the "cost" of the total path length from origin node $i$ to destination node $j$ using hubs $k$ and $m$, respectively, such that $c_{i j}^{k m}=\eta d_{i k}+\alpha d_{k m}+\delta d_{j m} \forall i, j \in N$ and $\forall k, m \in H . d_{i j}$ represents distance, time, etc. from origin $i$ to destination $j, \forall i, j \in N$ and it satisfies triangular inequality. Since the graph is complete, at most two hubs are used for each $O-D$ pair. For the interhub connection, 
the cost of the distance between the two hubs $k$ and $m$ is discounted by $\alpha$. $\eta$ and $\delta$ are the transportation factors for collection from an origin to a hub and for distribution from a hub to a destination, where generally $\alpha \leq \eta$ and $\alpha \leq \delta$. $\beta_{i j}$ is the maximum allowable service cost (or coverage distance) for each $O-D$ pair and $p$ is the number of hubs to be located. For binary coverage, a new binary parameter is defined, $a_{i j}^{k m}$, to decide whether an $O-D$ pair $(i-j)$ is covered by using hubs $k$ and $m$, respectively, or not:

$a_{i j}^{k m}=\left\{\begin{array}{ll}1 & \text { if } c_{i j}^{k m} \leq \beta_{i j} \\ 0 & \text { otherwise }\end{array} \quad \forall i, j \in N, \forall k, m \in H\right.$

For the partial coverage case, all parameters are the same except for $a_{i j}^{k m}$. We define a new parameter for coverage, $b_{i j}^{k m}$, and a new parameter for the upper bound, $\gamma_{i j}$, that is, for the service level that can be partially provided. Then, the coverage is defined as follows:

$b_{i j}^{k m}=\left\{\begin{array}{ll}1 & \text { if } c_{i j}^{k m} \leq \beta_{i j} \\ f\left(c_{i j}^{k m}\right) & \text { if } \beta_{i j} \leq c_{i j}^{k m} \leq \gamma_{i j} \\ 0 & \text { otherwise }\end{array} \quad \forall i, j \in N, \forall k, m \in H\right.$

where $f$ is any nonincreasing decay function and the range of the function $f$ is $(0,1)$.

The first linear formulation for SApHMCP, given by Campbell [6], keeps track of the route for each $O-D$ pair. The formulation is as follows:

Campbel [6] max $\sum_{i \in N j \in N k \in H m \in H} \sum_{i j} a_{i j}^{k m} w_{i j} Y_{i j k m}$

s.t.

$\sum_{k \in H} H_{k}=p$

$\sum_{k \in H} \sum_{m \in H} Y_{i j k m}=1 \quad \forall i, j \in N$

$X_{i k} \leq H_{k} \quad \forall i \in N, k \in H$

$\sum_{j \in N m \in H} \sum_{i j}\left(w_{i j k m}+w_{j i} Y_{j i m k}\right)=\sum_{j \in N}\left(w_{i j}+w_{j i}\right) X_{i k} \quad \forall i \in N, k \in H$

$H_{k} \in\{0,1\} \quad \forall k \in H$

$0 \leq Y_{i j k m} \leq 1 \quad \forall i, j \in N, \forall k, m \in H$

$X_{i k} \in\{0,1\} \quad \forall i \in N, k \in H$

The binary variable $H_{k}$ takes 1 if a hub is located at node $k$ and 0 otherwise. $X_{i k}$ takes 1 if node $i$ is assigned to a hub located at node $k$ and 0 otherwise. $Y_{i j k m}$ is the fraction of coverage from origin node $i$ to destination node $j$ using the hubs located at nodes $k$ and $m$, respectively. The objective function maximizes the covered demand of all $O-D$ pairs. Constraint (2.3) guarantees that exactly $p$ hubs are opened. Constraint (2.4) assures that the flow for every $O-D$ pair is routed via some hub pair. Constraint (2.5) satisfies that node $i$ can be assigned to node $k$ if $k$ is a hub. Constraint (2.6) guarantees the single allocation of each node using flow balance equality. Constraints (2.7)-(2.9) are for the domain restrictions.

The second formulation, which is similar to the formulation in [6], is proposed by Hwang and Lee [18]. The main difference is the constraint that satisfies the single allocation: instead of (2.6), the authors guarantee the single assignment with constraint (2.11). The other difference is that the $Y_{i j k m}$ variable is defined as a binary variable and $H_{k}$ is not used. The formulation is

Hwang and Lee [18] max (2.2)

s.t. (2.9)

$$
\begin{gathered}
X_{i k} \leq X_{k k} \quad \forall i \in N, k \in H \\
\sum_{k \in H} X_{i k}=1 \quad \forall i \in N \\
\sum_{k \in H} X_{k k}=p \\
2 Y_{i j k m} \leq X_{i k}+X_{j m} \quad \forall i, j \in N, \forall k, m \in H \\
Y_{i j k m} \in\{0,1\} \quad \forall i, j \in N, \forall k, m \in H
\end{gathered}
$$

Although in both papers only binary coverage is defined, the formulations are also applicable to partial coverage by simply replacing $a_{i j}^{k m}$ with $b_{i j}^{k m}$ in the formulations.

We now propose a novel formulation for the $p$-hub maximal covering problem. The new formulation does not need to keep track of routes, so we do not need the four-indexed decision variables. The decision variables for assigning the demand nodes are adequate to calculate the fraction of coverage of each $O-D$ pairs. The proposed formulation for SAPHMCP is as follows:

$$
\text { P\&K-S max } \sum_{i \in N j \in N} \sum_{i j} w_{i j}
$$

s.t. $\quad(2.9)-(2.12)$

$$
Z_{i j} \leq \sum_{k \in H} a_{i j}^{k m} X_{i k}+\lambda_{i j}\left(1-X_{j m}\right) \quad \forall i, j \in N, m \in H
$$

$$
Z_{i j} \geq 0 \quad \forall i, j \in N
$$

The decision variable $X_{i k}$ is the same as in previous formulations: it takes 1 if node $i$ is assigned to a hub located at node $k$, and 0 otherwise. $Z_{i j}$ is the fraction of flow routed from origin node $i$ to destination node $j$ that is covered. The aim of the objective function is to maximize the covered demands between $O-D$ pairs. Constraints (2.9)-(2.12) are the standard hub covering assignment constraints, given in the previous formulation. Constraint (2.15) calculates the fraction of flow between $O-D$ pairs $i-j$ that is covered with the correct allocation of $X_{i k}$ (origin node $i$ to hub $k$ ) and $X_{j m}$ (destination node $j$ to hub $m$ ). To tighten the constraint, we utilize $\lambda_{i j}=\max _{k, m \in H} a_{i j}^{k m}$. Due to constraint (2.11), only one $m$, say $m^{\prime}$, can be $1\left(X_{j m^{\prime}}=1\right)$. So, constraint (2.15) reduces to either $Z_{i j} \leq \sum_{k \in H} a_{i j}^{k m}$ $X_{i k}+\lambda_{i j}$ for $X_{j m}=0 \forall m \neq m^{\prime}$ or $Z_{i j} \leq \sum_{k \in H} a_{i j}^{k m^{\prime}} X_{i k}$ for $X_{j m^{\prime}}=1$. Then, $X_{i k^{\prime}}=1$ such that $k^{\prime}=\operatorname{argmax}_{k \in H}\left\{a_{i j}^{k m}\right\}$ since the first inequality is redundant due to the definition of $\lambda_{i j}$. Finally, constraint (2.16) is the non-negativity of the coverage variable.

The new formulation is readily applicable to the partial coverage case by replacing $a_{i j}^{k m}$ with $b_{i j}^{k m}$. Obviously, the proposed model has fewer variables and constraints than the previous formulations.

\subsection{Strengthening the formulation}

First, we observe that the following inequality is valid for the single allocation $p$-hub maximal covering problem since $Z_{i j}$ stands for the fraction of flow that is covered between $O-D$ pairs. Therefore, the maximum value it can take is $\lambda_{i j}$.

$Z_{i j} \leq \lambda_{i j} \quad \forall i, j \in N$

We also derive several valid inequalities for SApHMCP to strengthen the formulation.

Proposition 2.1. Inequality

$Z_{i j} \geq \sum_{k \in H} a_{i j}^{k m} X_{i k}+\left(X_{j m}-1\right) \quad \forall i, j \in N, m \in H$

is valid for $P \& K-S$.

Proof. Due to constraint (2.11), $\exists$ a node $s$ such that $X_{i s}=1$ and $X_{i t}=0 \forall t \neq s$. Thus, $\sum_{k \in H} a_{i j}^{k m} X_{i k}=a_{i j}^{s m}$. Therefore, if destination 
node $j$ is assigned to hub $m\left(X_{j m}=1\right)$, then (2.18) simplifies to $Z_{i j} \geq$ $a_{i j}^{s m}$ and is the correct coverage fraction via hubs $s$ and $m$. If $X_{j m}=0$, the inequality becomes $Z_{i j} \geq a_{i j}^{s m}-1$ and is a redundant constraint since $a_{i j}^{s m} \leq 1 \forall i, j, m$.

Proposition 2.2. The following inequality is valid for P\&K-S:

$Z_{i j} \leq \sum_{m \in H}\left(a_{i j}^{k m}-\lambda_{i j}\right) X_{i k}+\lambda_{i j} \quad \forall i, j \in N, k \in H$

Proof. If $X_{i k}=0$, (2.19) becomes $Z_{i j} \leq \lambda_{i j}$, that is, the valid inequality (2.17). Due to constraint (2.11), $\exists$ a node $s$ such that $X_{i s}=1$, and then the constraint simplifies to $Z_{i j} \leq \sum_{m \in H}\left(a_{i j}^{s m}\right)$. Since in the optimum solution, $Z_{i j}=a_{i j}^{s n}$ for a hub $n \in H$, (2.19) is valid since $a_{i j}^{s n} \leq \sum_{m \in H} a_{i j}^{s m} \forall i, j, n$.

The propositions are also valid for partial coverage by replacing $a_{i j}^{k m}$ with $b_{i j}^{k m}$.

\section{Multiple allocation p-Hub maximal covering problem (MAрHMCP)}

\subsection{Model development of MApHMCP}

In the multiple allocation $p$-hub maximal covering problem, any demand node can be allocated to several hubs. Campbell [6] poses the first linear formulation of this problem:

$$
\begin{aligned}
& \text { Campbell [6] max } \\
& \text { s.t. (2.3), (2.4), (2.7), (2.8) } \\
& Y_{i j k m} \leq H_{k} \quad \forall i, j \in N, \quad \forall k, m \in H \\
& Y_{i j k m} \leq H_{m} \quad \forall i, j \in N, \quad \forall k, m \in H
\end{aligned}
$$

The objectives and constraints are similar to the formulation for the single allocation version given in [6]. Since an assignment to exactly one hub is not necessary, the assignment variable $X_{i k}$ is not used in the multiple allocation version. For guaranteeing that only hubs are used for the route assignments of $O-D$ pairs, constraints (3.1) and (3.2) are added to the formulation. This formulation can easily be applied to the partial coverage case by changing the coverage parameter $a_{i j}^{k m}$ to $b_{i j}^{k m}$.

Weng et al. [31] have a different formulation for the same problem. They neither keep track of the routes for the $O-D$ pairs nor the assignments of the demand nodes to hubs. They only calculate the coverage for $O-D$ pairs that can be covered with located hubs. The formulation is

$$
\begin{aligned}
& \text { Weng et al. [31] } \max \sum_{i \in N j \in N} \sum_{i j} w_{i j} \\
& \text { s.t. } \quad(2.3),(2.7) \\
& \quad U_{i j} \leq \sum_{k \in H m \in H} \sum_{i j}^{k m} W_{k m} \quad \forall i, j \in N \\
& H_{k}+H_{m} \geq 2 W_{k m} \quad \forall k, m \in H \\
& W_{k m} \in\{0,1\} \quad \forall k, m \in H \quad \text { and } U_{i j} \in\{0,1\} \quad \forall i, j \in N
\end{aligned}
$$

The decision variable $U_{i j}$ takes 1 if the $O-D$ pair $i-j$ is covered by located hubs and 0 otherwise. They define $H_{k}$ to be 1 if a hub is located at node $k$, and 0 otherwise. $W_{k m}$ is 1 if both nodes $k$ and $m$ are selected as hubs and 0 otherwise. The objective function maximizes the covered demand of $O-D$ pairs by located hubs. Constraint (3.4) assures that $O-D$ pair $i-j$ can be covered if two hubs (or the same hub, i.e. $W_{k k}$ ) cover the path. Constraint (3.5) ensures if that $W_{k m}$ can be 1 only if $H_{k}$ and $H_{m}$ are 1 . Constraint (3.6) is for the integrality of the decision variables. Since it does not include the path for each $O-D$ pairs, the formulation has $O\left(n^{2}\right)$ constraints and $O\left(n^{2}\right)$ variables.
We remark here that, even though it is not given in [31], $U_{i j}$, which is defined as a binary variable in [31], can be relaxed as $U_{i j} \leq 1$ without losing optimality.

This formulation is not applicable to the partial coverage model because the formulation might not calculate the correct coverage of $O-D$ pairs $i-j$ for that case. Even with the relaxed definition of $U_{i j}$, there is a possibility of calculating an incorrect coverage of $O-D$ pairs. This problem can be observed from the following example: Consider a network on five nodes and let $N=\{1,2\}$ and $H=\{3,4,5\}$. Let the coverage values for the nodes in the set $N$ be $b_{12}^{34}=b_{21}^{43}=0.75$ and $b_{12}^{35}=b_{21}^{53}=0.5$, and let the rest of the values be equal to 0 . If the formulation in [31] is used to solve MApHMCP with partial coverage for $p=3$, from constraint (3.4), we obtain $U_{12} \leq 1.25$ and $U_{21} \leq 1.25$. Due to constraint (3.6), they both take the value 1 . However, $U_{12}$ and $U_{21}$ should be equal to 0.75 . Thus, for the multiple allocation version of the $p$-hub maximal covering problem for the partial coverage case, the model in Campbell [6] is the only formulation from the previous literature that can be used.

We now propose a new formulation for MApHMCP that is readily applicable to the partial coverage case. The notion of this formulation is different than both of the formulations given in the literature. Let $X_{i j k}$ be 1 if the first hub of the $O-D$ pair $i-j$ is $k$, and $Y_{i j m}$ be 1 if the second hub of the $O-D$ pair $i-j$ is $m$. $H_{k}$ takes 1 if node $k$ is selected as a hub, otherwise it is zero. $Z_{i j}$ is the fraction of flow routed from origin node $i$ to destination node $j$ that is covered. The proposed formulation is

$$
\begin{aligned}
& \text { P\&K-M max (2.14) } \\
& \text { s.t. } \quad(2.3),(2.7),(2.16) \\
& Z_{i j} \leq \sum_{k \in H} a_{i j}^{k m} X_{i j k}+\lambda_{i j}\left(1-Y_{i j m}\right) \quad \forall i, j \in N, m \in H \\
& Z_{i j} \leq \sum_{m \in H} a_{i j}^{k m} Y_{i j m}+\lambda_{i j}\left(1-X_{i j k}\right) \quad \forall i, j \in N, k \in H \\
& \sum_{k \in H} X_{i j k}=1 \quad \forall i, j \in N \\
& \sum_{m \in H} Y_{i j m}=1 \quad \forall i, j \in N \\
& X_{i j k} \leq H_{k} \quad \forall i, j \in N, k \in H \\
& Y_{i j m} \leq H_{m} \quad \forall i, j \in N, m \in H \\
& X_{i j k} \in\{0,1\} \quad \forall i, j \in N, k \in H \\
& Y_{i j m} \in\{0,1\} \quad \forall i, j \in N, m \in H
\end{aligned}
$$

The objective function maximizes the covered demand of all $i-j$ pairs. Constraints (3.7) and (3.8) calculate the fraction of flow between $O-D$ pairs $i-j$ that is covered using the correct route allocations of $X_{i j k}$ and $Y_{i j m}$. Constraint (3.9) guarantees that each path from origin node $i$ to destination node $j$ can be assigned to only one hub as the first hub. Similarly, constraint (3.10) satisfies that the same route can be assigned to only one hub as the second hub. Constraints (3.11) and (3.12) satisfy that the path $i-j$ can be assigned to nodes $k$ and $m$ only if $k$ and $m$ are hubs, respectively. Constraints (3.13) and (3.14) are the domain constraints.

\subsection{Strengthening the formulation}

Similar notions for developing the valid inequalities used in Section 2.2 can be also used for the multiple allocation version of the problem. The first inequality (2.17) is also valid for P\&K-M: 
Proposition 3.1. The following inequality is valid for P\&K-M.

$Z_{i j} \geq \sum_{k \in H} a_{i j}^{k m} X_{i j k}+\left(Y_{i j m}-1\right) \quad \forall i, j \in N, m \in H$

Proof. Due to constraint (3.9), $\exists s$ such that $X_{i j s}=1$ and $X_{i j t}=0 \forall$ $t \neq s$. Thus, $\sum_{k \in H} a_{i j}^{k m} X_{i j k}=a_{i j}^{s m}$. Then, if $Y_{i j m}=1$, (3.15) simplifies to $Z_{i j} \geq a_{i j}^{s m}$, which is the coverage of flow between $O-D$ pairs via hubs $s$ and $m$. If $Y_{i j m}=0$, then $Z_{i j} \geq a_{i j}^{s m}-1$ and it is a redundant constraint since $a_{i j}^{s m} \leq 1 \forall i, j, m$, given that the range of function $f$ is $(0,1)$.

Proposition 3.2. The following inequality is valid for P\&K-M:

$Z_{i j} \leq \sum_{m \in H}\left(a_{i j}^{k m}-\lambda_{i j}\right) X_{i j k}+\lambda_{i j} \quad \forall i, j \in N, k \in H$

Proof. Inequality (3.16) is similar to inequality (2.19) proposed for P\&K-S. So, the same proof also holds for inequality (3.16), with $X_{i j k}$ replacing the $X_{i k}$ in (2.19). The inequalities are also valid for the partial coverage case by replacing $a_{i j}^{k m}$ with $b_{i j}^{k m}$.

\section{Computational complexity}

In this section, we show that both SApHMCP and MApHMCP are NP-Hard. The second result is also known from [31] in considering every $O-D$ pair as a single node. We present that our proof for the NP-Hardness of SApHMCP can also be used for MApHMCP. We prove the NP-Hardness of the problems with binary coverage, and thus the NP-Hardness of the problems with partial coverage follows. We also prove that SApHMCP is NP-Hard even if the hub locations are known.

Proposition 4.1. SApHMCP with binary coverage is NP-Complete even if $\alpha=0, \delta=0$.

Proof. To show the NP-Completeness of the problem, we reduce the maximum coverage problem (MCP) to SApHMCP in polynomial time with the following instance:

The decision version of SApHMCP is as follows: Is there a set of vertices $H \subseteq V$ and $|H|=p$ with an assignment vector $u$, where $u_{i} \in H$, such that $\sum_{i, j \in S} w_{i j} \geq T$, where $S$ is a set of vertices with property $\eta d_{i u_{i}}+\alpha d_{u_{i} u_{j}}+\delta d_{u_{j} j} \leq \beta \forall i, j \in S$ and $u_{i}, u_{j} \in H$ ? The instance for the problem is given as $G=(V, E), \eta, \alpha, \delta \geq 0, p \leq|V| . \quad \beta$ represents the coverage distance, weight $w_{i j} \forall i, j \in V$, distance $d_{i j} \forall i, j \in V$ and $T \geq 0$.
Similarly, the decision version of MCP can be stated as follows: Is there a set of vertices $H^{\prime} \subseteq V^{\prime}$ and $\left|H^{\prime}\right|=p$ such that $\sum_{i: \exists j \in H^{\prime}}$ and $d_{i j} \leq \beta w_{i} \geq T^{\prime}$ ? The instance for MCP is also given as $G^{\prime}=\left(V^{\prime}, E^{\prime}\right)$, $p \leq\left|V^{\prime}\right|, \beta$ is for coverage distance, weight $w_{i} \forall i \in V^{\prime}$, distance $d_{i j} \forall i, j \in V^{\prime}$ and $T^{\prime} \geq 0$.

For MCP, consider an arbitrary instance of the graph $G^{\prime}$, where vertices $V^{\prime}$ denote the set of customers and potential sites for the facilities. Let $w_{i}$ be the demand of customer $i$, and if $d_{i j} \leq \beta$ for $i \in V^{\prime}, j \in H^{\prime}$, then $w_{i}$ is covered. At most $p$ facilities can be located at the potential sites, and this problem (MCP) is known to be NP-Hard [24]. Now consider an instance of SApHMCP with the following data set: $G=G^{\prime}$ and the flow $\forall i, j \in V$ is $w_{i j}=w_{i} /\left(\left|V^{\prime}\right|-1\right)$ for $i \neq j$ and $w_{i i}=0 . \alpha=\delta=0$ and hubs can be opened in at most $p$ locations. Then, the two problems are equivalent, because MCP has a solution, with at most $p$ facilities satisfying $\sum_{i: \exists j \in H^{\prime}}$ and $d_{i j} \leq \beta w_{i} \geq T^{\prime}$ if and only if SApHMCP has a solution with at most $p$ hubs with $\sum_{i, j \in S} w_{i j} \geq T$, where $S$ is a set of vertices with property $\eta d_{i u_{i}}+\alpha d_{u_{i} u_{j}}+\delta d_{u_{j} j} \leq \beta \forall i, j \in S$ and $u_{i}, u_{j} \in H$. From the solution of MCP, a vector $u$ can be generated as $k=\operatorname{argmin}_{l \in H^{\prime}} d_{i l}$ and $u_{i}=k \forall i \in V^{\prime}$, and from the solution of SApHMCP, a solution for MCP can be obtained: if $d_{i u_{i}} \leq \beta$ then $w_{i}$ is covered. So, SApHMCP is NP-Complete.

Proposition 4.2. MApHMCP with binary coverage is NP-Complete even if $\alpha=0, \delta=0$.

Proof. To show the NP-Completeness of the problem, the same reduction in Proposition 4.1 can be used. To generate the assignments, let $R_{i}=\left\{l \in H^{\prime}: d_{i l} \leq \beta\right\}$. Then, $u_{i}=k \forall k \in R_{i}$ and $\forall i \in V^{\prime}$, which becomes a solution of MApHMCP. From this solution, a solution of MCP can be generated: if $d_{i u_{i}} \leq \beta$ holds at least one element of $u_{i}$, then $w_{i}$ is covered. Thus, MApHMCP is NPComplete.

Alternatively, we can prove the NP-Hardness of the problems by showing that a specific instance of them is equivalent to the $p$-hub center problem. Let an instance of SApHMCP be such that $\eta, \alpha, \delta \leq 1$, distance is $d_{i j}$ and weight is $w_{i j} \forall i, j \in V$. For coverage distance, let $\beta=3 \max _{(i, j) \in E} d_{i j}$ and $T=\sum_{(i, j) \in E} w_{i j}$. With these parameter settings, the condition $\sum_{i, j \in S} w_{i j} \geq T$, where $S$ is as given above, is directly satisfied, and thus SAPHMCP is equivalent to the single allocation $p$-hub center problem. Therefore, solving SApHMCP with that instance will be as hard as solving the $p$-hub center problem with that instance. Since the single allocation

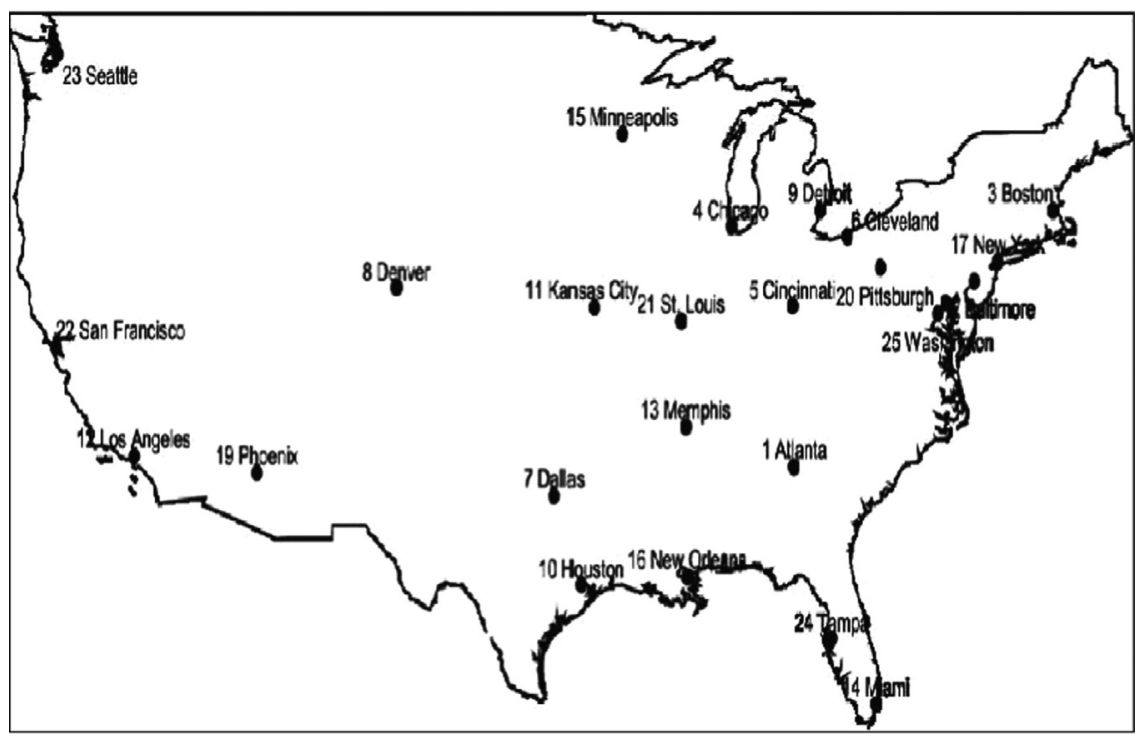

Fig. 1. Locations of demand nodes and possible hub locations for the CAB data set. 
p-hub center problem is in NP-Hard [20], SApHMCP is also in NP.

A similar conversion can be easily applied for the proof of the NP-Hardness of the multiple allocation version of the $p$-hub maximal covering problem. Since the multiple allocation version of the p-hub center problem is proven NP-Hard in Ernst et al. [14], using the same data set given above, MApHMCP reduces to the multiple allocation $p$-hub center problem.

As a special case of the $p$-hub maximal covering problem, we also discuss the complexity of the allocation problems. The allocation problem is the problem of determining the assignments of nonhub nodes to hub(s) whose locations are fixed and known in advance.

The allocation problem of the multiple assignment $p$-hub maximal covering problem is polynomially solvable by solving the $|N|^{2}$ shortest path for each $O-D$ pair, where $|N|$ is the cardinality of the number of nonhub nodes. However, the allocation problem of SApHMCP is in NP since a special instance of the problem is equivalent to the allocation problem of the $p$-hub center problem, whose NP-Hardness is also proven by Ernst et al. [14].

Proposition 4.3. The allocation problem of SAPHMCP is NPComplete.

Proof. The decision version of SApHMCP can be expressed as follows: Is there an assignment vector $u$, where $u_{i} \in H$, such that $\sum_{i, j \in S} w_{i j} \geq T$, where $S$ is a set of vertices with property

Table 1

Solutions of the $p$-hub center problem for the CAB data set for $\alpha=0.2,0.4,0.6$, 0.8 and $p=2-5[21]$.

\begin{tabular}{lll}
\hline$\alpha$ & $p$ & Obj. value $\left(\beta^{\prime}\right)$ \\
\hline 0.2 & 2 & 2136 \\
& 3 & 1913 \\
& 4 & 1617 \\
& 5 & 1346 \\
0.4 & 2 & 2401 \\
& 3 & 2099 \\
& 4 & 1881 \\
& 5 & 1597 \\
0.6 & 5 & 2557 \\
& 2 & 2336 \\
& 3 & 2184 \\
& 4 & 2002 \\
0.8 & 5 & 2713 \\
& 2 & 2552 \\
& 3 & 2457 \\
& 4 & 2307 \\
\hline
\end{tabular}

$\eta d_{i u_{i}}+\alpha d_{u_{i} u_{j}}+\delta d_{u_{j} j} \leq \beta \forall i, j \in S$ and $u_{i}, u_{j} \in H$ ? Similarly, the decision version of the allocation problem of the hub center problem can be given as: Is there an assignment vector $u$, where $u_{i} \in H$ such that $\eta d_{i u_{i}}+\alpha d_{u_{i} u_{j}}+\delta d_{u_{j} j} \leq \beta \forall i, j \in V$ and $u_{i}, u_{j} \in H$ ?

Let an instance of SApHMCP be such that $\eta, \alpha, \delta \leq 1$, distance is $d_{i j}$ and weight is $w_{i j} \forall i, j \in V$. For coverage distance, let $\beta=3 \max _{(i, j) \in E} d_{i j}$ and $T=\sum_{(i, j) \in E} w_{i j}$. The decision version of the allocation problem of SApHMCP can be modified with $\sum_{i, j \in S}$ $w_{i j} \geq T$, as done in the alternative proof of the NP-Hardness of SApHMCP. Then the decision version of the allocation problem of SApHMCP is equivalent to the decision version of the allocation problem of the $p$-hub center problem. Since the allocation problem of the $p$-hub center is NP-Hard [14], the allocation problem of SApHMCP is proven to be NP-Hard.

\section{Computational results for SApHMCP}

In this section, we first explain the data sets and the parameters that are used for computational test. We then test the combination of valid inequalities for the proposed formulation. For SApHMCP, we compare the results of the new formulation with the existing ones explained in Section 2. We also discuss the effect of allowing partial coverage to the solution time, coverage percentage, hub locations and allocation of nonhub nodes. For this purpose, we compare the solutions of the proposed formulation with binary and partial coverage types for different instances.

\subsection{Data generation}

We tested the proposed formulations with US Civil Aeronautics Board (CAB) and Turkish network (TR) data sets. O'Kelly [26] introduces the $C A B$ data sets based on airline passenger transportation between 25 US cities. All cities are considered possible hub locations (Fig. 1).

We used the distance matrix as in the original data set, and we scaled the flow to 100 for testing the formulations. For the maximum service distance, we generated parameter $(\beta)$ by using the results of the $p$-hub center problem, $\beta^{\prime}$, given in [21]. The results of the $p$-hub center problem for different $\alpha$ and $p$ values are presented in Table 1.

We used $\eta=\delta=1$ in all the computational analyses, and the parameter $a_{i j}^{k m}$ is defined as follows:

$a_{i j}^{k m}=\left\{\begin{array}{ll}1 & \text { if } c_{i j}^{k m} \leq 0.75 \beta^{\prime} \\ 0 & \text { otherwise }\end{array} \quad \forall i, j \in N \quad\right.$ and $\quad \forall k, m \in H$

For partial coverage, we use the same values of parameters $\alpha, p$ and $\beta$ that are used in the binary coverage. We adapt the definition of

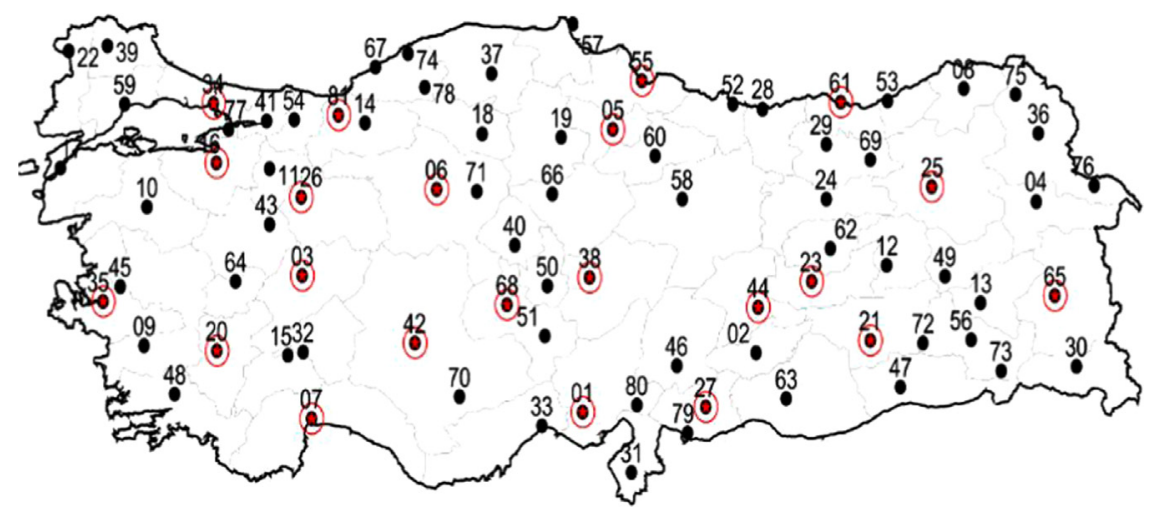

Fig. 2. Locations of demand nodes and hub locations for the TR data set. 
$b_{i j}^{k m}$ to the partial coverage with a stepwise function:

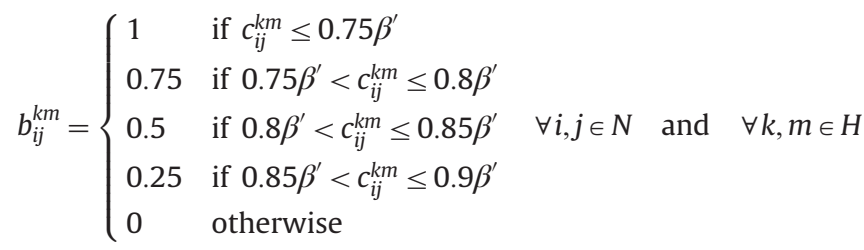

We also used a relatively larger data set, TR, to analyze the performance of the formulations. The TR data set compromises 81 demand nodes (cities), of which 22 are selected as potential hub locations. These 22 are among the most populated and industrialized cities in Turkey [29,32] and are shown in Fig. 2 with circles. For the computational analysis we varied $p$ between $5,10,15$ and 20.

We used travel times for calculating the parameters and we assumed that travel time is directly proportional to distance. For binary coverage, we considered a half-day service limit:

$a_{i j}^{k m}=\left\{\begin{array}{ll}1 & \text { if } c_{i j}^{k m} \leq 12 \mathrm{~h} \\ 0 & \text { otherwise }\end{array} \quad \forall i, j \in N \quad\right.$ and $\quad \forall k, m \in H$

where $d_{i j}$ is the travel time between nodes $i$ and $j$. For the partial coverage parameter setting, we again used a stepwise function with the upper bound $\gamma=24 \mathrm{~h}$, that is, beyond $24 \mathrm{~h}$ no path can be covered even partially.

$b_{i j}^{k m}=\left\{\begin{array}{ll}1 & \text { if } c_{i j}^{k m} \leq 12 \mathrm{~h} \\ 0.75 & \text { if } 12 \mathrm{~h}<c_{i j}^{k m} \leq 16 \mathrm{~h} \\ 0.5 & \text { if } 16 \mathrm{~h}<c_{i j}^{k m} \leq 20 \mathrm{~h} \\ 0.25 & \text { if } 20 \mathrm{~h}<c_{i j}^{k m} \leq 24 \mathrm{~h} \\ 0 & \text { otherwise }\end{array} \quad \forall i, j \in N\right.$ and $\quad \forall k, m \in H$

For the economies of scale parameter, we used estimations from Turkish cargo companies, who calculated the time and cost savings

Table 2

Effect of valid inequalities on P\&K-S with binary coverage for the TR data set.

\begin{tabular}{|c|c|c|c|c|c|c|c|c|c|c|}
\hline$\alpha$ & $p$ & & No valid ineq & $(2.17)$ & $(2.18)$ & $(2.19)$ & (2.18), (2.19) & (2.17), (2.19) & (2.17), (2.18) & (2.17), (2.18), (2.19) \\
\hline \multirow[t]{2}{*}{0.8} & 5 & Best bn at root node (\%) & 84.93 & 84.93 & 84.93 & 82.53 & 82.53 & 82.53 & 84.93 & 82.53 \\
\hline & & $\mathrm{CPU}(\mathrm{sec})$ & 255.77 & 250.88 & 516.19 & 171.21 & 619.11 & 146.24 & 381.69 & 321.34 \\
\hline \multirow[t]{2}{*}{0.8} & 10 & Best bn at root note (\%) & 88.63 & 88.63 & 88.63 & 87.61 & 87.61 & 87.61 & 88.63 & 87.61 \\
\hline & & CPU $(\mathrm{sec})$ & 113.74 & 98.25 & 195.95 & 43.8 & 81.52 & 35.38 & 247.45 & 88.54 \\
\hline \multirow[t]{2}{*}{0.9} & 5 & Best bn at root note (\%) & 79.61 & 79.61 & 79.61 & 76.86 & 76.86 & 76.86 & 79.61 & 76.86 \\
\hline & & CPU (sec) & 200.69 & 193.81 & 355.86 & 145.01 & 220.24 & 112.31 & 329.21 & 226.36 \\
\hline \multirow[t]{2}{*}{0.9} & 10 & Best bn at root note (\%) & 83.29 & 83.29 & 83.29 & 82.08 & 82.08 & 82.08 & 83.29 & 82.08 \\
\hline & & CPU (sec) & 92.02 & 76.78 & 159.34 & 16.77 & 33.95 & 19.25 & 177.26 & 40.08 \\
\hline
\end{tabular}

Table 3

Effect of valid inequalities on the P\&K-S with partial coverage for the TR data set.

\begin{tabular}{|c|c|c|c|c|c|c|c|c|c|c|}
\hline$\alpha$ & $p$ & & No valid ineq & $(2.17)$ & $(2.18)$ & $(2.19)$ & (2.18), (2.19) & (2.17), (2.19) & (2.17), (2.18) & (2.17), (2.18), (2.19) \\
\hline \multirow[t]{2}{*}{0.8} & 5 & Best bn at root node (\%) & 168.80 & 97.11 & 168.80 & 97.11 & 97.11 & 97.11 & 97.11 & 97.11 \\
\hline & & Gap (\%) & 79.17 & 2.26 & 80.61 & 2.62 & 2.48 & 2.22 & 3.02 & 3.04 \\
\hline \multirow[t]{2}{*}{0.8} & 10 & Best bn at root node (\%) & 166.97 & 97.36 & 166.97 & 97.36 & 97.36 & 97.36 & 97.36 & 97.36 \\
\hline & & Gap (\%) & 71.32 & 0.33 & 74.75 & 0.44 & 0.41 & 0.23 & 0.46 & 0.46 \\
\hline \multirow[t]{3}{*}{0.9} & 5 & Best bn at root node (\%) & 167.16 & 95.83 & 167.16 & 95.83 & 95.83 & 95.83 & 95.83 & 95.83 \\
\hline & & Root node $(\%)$ & & & & & & & & \\
\hline & & Gap (\%) & 79.60 & 2.05 & 79.51 & 2.10 & 2.32 & 2.05 & 2.34 & 2.34 \\
\hline \multirow[t]{3}{*}{0.9} & 10 & Best bn at root node (\%) & 165.00 & 96.05 & 165.00 & 96.05 & 96.05 & 96.05 & 96.05 & 96.05 \\
\hline & & Root node (\%) & & & & & & & & \\
\hline & & Gap (\%) & 71.13 & 0.29 & 77.01 & 0.36 & 0.42 & 0.29 & 0.42 & 0.43 \\
\hline
\end{tabular}

Table 4

SApHMCP solutions with binary coverage for the CAB data set.

\begin{tabular}{|c|c|c|c|c|c|c|c|c|c|}
\hline \multirow[t]{2}{*}{$\alpha$} & \multirow[t]{2}{*}{$p$} & \multicolumn{2}{|l|}{ P\&K-S } & \multicolumn{3}{|l|}{ Campbell [6] } & \multicolumn{3}{|c|}{ Hwang and Lee [18] } \\
\hline & & Coverage (\%) & CPU (sec) & best int (\%) & CPU (sec)/opt.gap (\%) & bb at root (\%) & best int (\%) & CPU/opt.gap (\%) & bb at root (\%) \\
\hline \multirow[t]{4}{*}{0.6} & 2 & 90.01 & 8.93 & Opt & $2542.6 / 0.00$ & 100 & 79.24 & $>1 \mathrm{~h} / 11.97$ & 1189.93 \\
\hline & 3 & 91.91 & 3.7 & 90.57 & $>1 \mathrm{~h} / 1.46$ & 100 & 89.31 & $>1 \mathrm{~h} / 2.83$ & 1109.25 \\
\hline & 4 & 91.51 & 4.65 & 87.16 & $>1 \mathrm{~h} / 4.75$ & 100 & 89.83 & $>1 \mathrm{~h} / 1.84$ & 1036.36 \\
\hline & 5 & 88.38 & 3.28 & 86.32 & $>1 \mathrm{~h} / 2.33$ & 96.71 & 86.90 & $>1 \mathrm{~h} / 1.68$ & 945.55 \\
\hline \multirow[t]{4}{*}{0.8} & 2 & 87.79 & 1.06 & 80.46 & $>1 \mathrm{~h} / 8.35$ & 97.18 & 78.16 & $>1 \mathrm{~h} / 10.97$ & 1130.56 \\
\hline & 3 & 87.35 & 2.16 & 81.29 & $>1 \mathrm{~h} / 6.93$ & 92.72 & 83.70 & $>1 \mathrm{~h} / 4.17$ & 1086.78 \\
\hline & 4 & 87.30 & 4.36 & 82.56 & $>1 \mathrm{~h} / 5.43$ & 91.87 & 83.35 & $>1 \mathrm{~h} / 4.53$ & 1059.68 \\
\hline & 5 & 86.23 & 2.02 & 85.12 & $>1 \mathrm{~h} / 1.29$ & 90.85 & 83.43 & $>1 \mathrm{~h} / 3.25$ & 1000.70 \\
\hline
\end{tabular}


Table 5

SApHMCP solutions with partial coverage for the CAB data set.

\begin{tabular}{|c|c|c|c|c|c|c|c|c|c|}
\hline \multirow[t]{2}{*}{$\alpha$} & \multirow[t]{2}{*}{$p$} & \multicolumn{2}{|l|}{ P\&K-S } & \multicolumn{3}{|l|}{ Campbell [6] } & \multicolumn{3}{|c|}{ Hwang and Lee [18] } \\
\hline & & Coverage (\%) & CPU (sec) & Best int (\%) & CPU (sec)/opt. gap (\%) & bb at root (\%) & best int (\%) & CPU/opt. gap (\%) & bb at root (\%) \\
\hline \multirow[t]{4}{*}{0.6} & 2 & 93.47 & 29.05 & Opt & $3315.73 / 0.0$ & 100 & Opt & $>1 \mathrm{~h} / 0.00$ & 1376.07 \\
\hline & 3 & 94.02 & 38.76 & 92.65 & $>1 \mathrm{~h} / 1.45$ & 100 & 93.94 & $>1 \mathrm{~h} / 0.08$ & 1284.49 \\
\hline & 4 & 94.62 & 43.84 & 88.20 & $>1 \mathrm{~h} / 6.79$ & 100 & 92.77 & $>1 \mathrm{~h} / 1.95$ & 1211.82 \\
\hline & 5 & 93.0 & 131.1 & 91.47 & $>1 \mathrm{~h} / 1.65$ & 99.07 & 90.08 & $>1 \mathrm{~h} / 3.14$ & 1098.50 \\
\hline \multirow[t]{4}{*}{0.8} & 2 & 91.83 & 26.95 & 90.68 & $>1 \mathrm{~h} / 1.24$ & 99.28 & 84.52 & $>1 \mathrm{~h} / 7.95$ & 1322.16 \\
\hline & 3 & 90.86 & 50.27 & 85.81 & $>1 \mathrm{~h} / 5.56$ & 97.46 & 89.19 & $>1 \mathrm{~h} / 1.83$ & 1261.81 \\
\hline & 4 & 90.87 & 116.57 & 87.52 & $>1 \mathrm{~h} / 3.69$ & 97.03 & 88.88 & $>1 \mathrm{~h} / 2.19$ & 1236.92 \\
\hline & 5 & 89.29 & 132.42 & 86.34 & $>1 \mathrm{~h} / 3.30$ & 93.96 & 87.01 & $>1 \mathrm{~h} / 2.55$ & 1157.85 \\
\hline
\end{tabular}

Table 6

Solutions of P\&K-S for binary and partial coverage types for the TR data set.

\begin{tabular}{|c|c|c|c|c|c|}
\hline \multirow[t]{2}{*}{$\alpha$} & \multirow[t]{2}{*}{$p$} & \multicolumn{2}{|l|}{ Binary } & \multicolumn{2}{|l|}{ Partial } \\
\hline & & Coverage (\%) & CPU (sec) & Coverage (\%)/best int (\%) & CPU (sec)/gap (\%) \\
\hline \multirow[t]{4}{*}{0.8} & 5 & 78.83 & 146.24 & $(94.59)$ & $>2 \mathrm{~h} / 2.26$ \\
\hline & 10 & 86.74 & 35.38 & 96.68 & 4850.03 \\
\hline & 15 & 89.48 & 2.53 & 97.37 & 367.06 \\
\hline & 20 & 90.02 & 2.1 & 97.51 & 10.11 \\
\hline \multirow[t]{4}{*}{0.9} & 5 & 73.78 & 112.31 & $(93.33)$ & $>2 \mathrm{~h} / 2.05$ \\
\hline & 10 & 81.71 & 19.25 & 95.43 & 4342.87 \\
\hline & 15 & 83.54 & 3.88 & 95.88 & 1040.17 \\
\hline & 20 & 84.38 & 0.72 & 96.09 & 211.99 \\
\hline
\end{tabular}

due to flow consolidation as $10 \%$ and $20 \%$, respectively [32]. We considered both savings and $\alpha$ was taken as 0.8 and 0.9 for interhub connections.

Since the data sets used in this paper ( $C A B$ and TR) have symmetric distance matrices, the solution time can be improved by reducing the number of constraints. So, constraint (2.15) is substituted with

$Z_{i j} \leq \sum_{k \in H} a_{i j}^{k m} X_{i k}+\lambda_{i j}\left(1-X_{j m}\right) \quad \forall i, j \in N: i \leq j, \quad \forall m \in H$

Similarly, $i \leq j$ is added to all the valid inequalities (2.17)-(2.19) given in Section 2.2. The objective function (2.14) can also be improved in a similar way, since $Z_{i j}=Z_{j i} \quad \forall i, j \in N$ :

$\max \sum_{i \in N} \sum_{j \in N: i \leq j}\left(w_{i j}+w_{j i}\right) Z_{i j}$

\subsection{Performance analysis of the proposed valid inequalities}

We first compare all the combinations of valid inequalities given in Section 2.2 for P\&K-S with the TR data set for both the binary and partial coverage types. All the computational results were made on a Linux environment with a 4xAMD Opteron Interlagos 16C 6282SE 2.6G 16 M 6400MT 96 GB RAM. Based on our preliminary analyses, we decided to use CPLEX 12.4 for single allocation and Gurobi 5.0.2 for multiple allocation.

In each multi-row in Tables 2 and 3, the first line lists the percentage of the best upper bound at the root node and the second line presents either the CPU time (if the solution time is under one hour) or the gap reported by the solver at the end of the one-hour time limit. Columns 4 through 11 correspond to all possible combinations of the valid inequalities. Our preliminary analyses show that for binary coverage, the lowest best bound at the root node is achieved when inequality
Table 7

Partially covered $O-D$ pairs for the CAB data set for the instance with $\alpha=0.2, p=4$.

\begin{tabular}{llllll}
\hline$(i, j)$ & $Z_{i j}=Z_{j i}$ & $(i, j)$ & $Z_{i j}=Z_{j i}$ & $(i, j)$ & $Z_{i j}=Z_{j i}$ \\
\hline$(1,23)$ & 0.5 & $(9,22)$ & 0.75 & $(11,23)$ & 0.75 \\
$(2,23)$ & 0.25 & $(10,14)$ & 0.5 & $(14,19)$ & 0.5 \\
$(8,14)$ & 0.75 & $(10,19)$ & 0.75 & $(14,22)$ & 0.5 \\
$(9,10)$ & 0.75 & $(10,22)$ & 0.75 & $(19,23)$ & 0.5 \\
$(9,19)$ & 0.75 & & & & \\
\hline
\end{tabular}

(2.19) is added to any of the combinations (columns 7-9, 11) (Table 2). The lowest solution times are obtained when inequalities (2.17) and (2.19) are used together, except for the last row (where the difference is about $2.5 \mathrm{~s}$ ). For partial coverage, the lowest percentage of best upper bounds at the root nodes are achieved with either (2.17) or (2.19) (or both) (Table 3). In terms of the gaps at the end of the one-hour time limit, the lowest gaps are obtained with inequalities (2.17) and (2.19). Due to the above findings, we decided to use valid inequalities (2.17) and (2.19) in the computational experiments for P\&K-S.

\subsection{Computational experiments for SApHMCP}

In this section, we compare the solutions of the new formulation with the existing ones explained in Section 2. For comparison purposes, although applicability to the partial coverage is not mentioned in the existing literature, in addition to binary coverage, we compared the results with the application of partial coverage. We tested the models with the CAB and TR data sets and used a time limit of one hour for the small data set (CAB) and two hours for the large data set (TR).

In all Tables 4-6 and 10-12, in the coverage(\%) column, we report the optimal solution as the percentage of total flow covered. The CPU column shows the solution time of those instances and the $b b$ at root(\%) column shows the best upper bound percentage that is obtained at the root node. If optimality is not verified within the time limit, we report the best int(\%), which is the corresponding coverage percentage for the best solution obtained at the end of the time limit. Last, in column opt. gap(\%), we report the gap between the best solution and the optimal solution, calculated as (coverage - best int)/coverage $\times 100$.

For the $C A B$ data set, since the gaps of the existing formulations reported by the solver are too high, we only report and compare the solutions for $\alpha=0.6$ and 0.8 (Tables 4 and 5). The results of the proposed formulation for the remaining instances are given in the Appendix Table A1. It is evident from Tables 4 and 5 that P\&K-S outperforms the existing formulations. The maximum solution time of the proposed formulation is about nine seconds, whereas the existing formulations usually must be terminated before solving due to the time limit. The results show that the most up to date formulation in the literature, given in [18], performs worse than the basic formulation [6] although the main purpose of the paper was not computational 

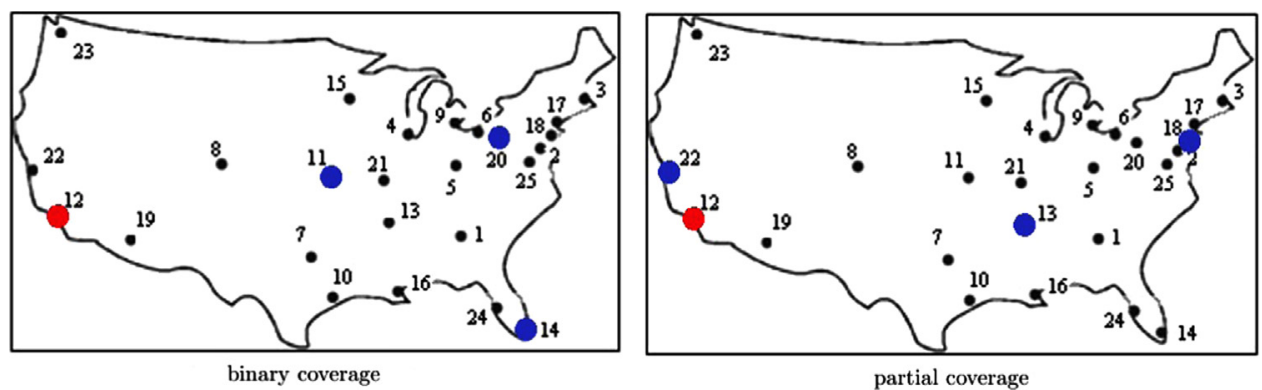

Fig. 3. Hub locations for single allocation for the instance $\alpha=0.6, p=4$ with both coverage types for the CAB data set.
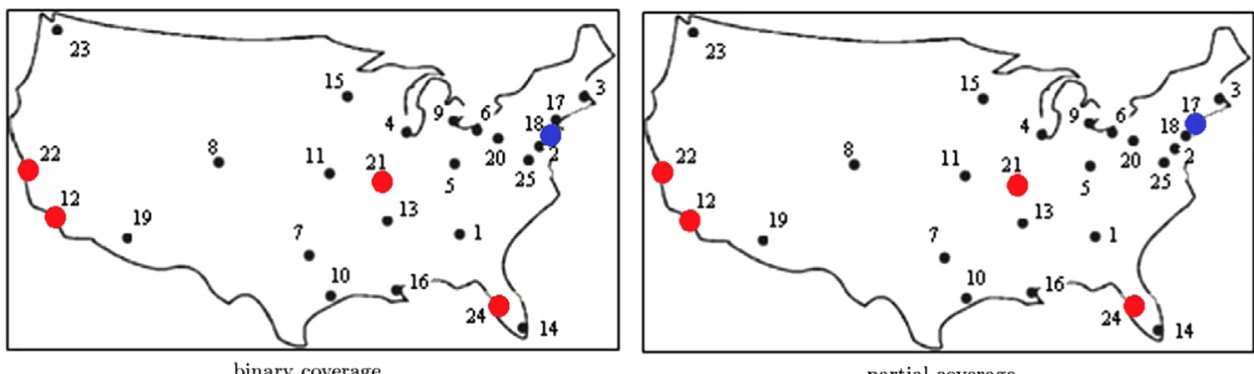

Fig. 4. Hub locations of hubs for single allocation for the instance $\alpha=0.4, p=5$ with both coverage types for the CAB data set.

Table 8

Effect of valid inequalities on P\&K-M with binary coverage for the TR data set.

\begin{tabular}{|c|c|c|c|c|c|c|c|c|c|c|}
\hline$\alpha$ & $p$ & & No valid ineq & $(2.17)$ & $(3.15)$ & $(3.16)$ & (3.15), (3.16) & (2.17), (3.16) & (2.17), (3.15) & (2.17), (3.15), (3.16) \\
\hline \multirow[t]{2}{*}{0.8} & \multirow[t]{2}{*}{5} & Best bn at root node (\%) & 90.17 & 90.17 & 90.17 & 90.14 & 90.14 & 90.14 & 90.17 & 90.14 \\
\hline & & Gap (\%) & 8.85 & 8.80 & 9.63 & 3.20 & 3.22 & 3.20 & 9.46 & 3.16 \\
\hline \multirow[t]{2}{*}{0.8} & \multirow[t]{2}{*}{10} & Best bn at root node (\%) & 90.29 & 90.29 & 90.29 & 90.27 & 90.27 & 90.27 & 90.29 & 90.27 \\
\hline & & Gap (\%) & 1.61 & 1.61 & 1.64 & 0.56 & 0.43 & 0.68 & 1.64 & 0.43 \\
\hline \multirow[t]{2}{*}{0.9} & \multirow[t]{2}{*}{5} & Best bn at root node (\%) & 85.19 & 85.19 & 85.19 & 85.17 & 85.17 & 85.17 & 85.19 & 85.17 \\
\hline & & Gap (\%) & 11.00 & 11.00 & 8.91 & 6.80 & 6.18 & 6.80 & 8.91 & 6.18 \\
\hline \multirow[t]{2}{*}{0.9} & \multirow[t]{2}{*}{10} & Best bn at root node (\%) & 85.26 & 85.26 & 85.26 & 85.25 & 85.25 & 85.25 & 85.26 & 85.25 \\
\hline & & $\mathrm{CPU}(\mathrm{h})$ & 0.70 & 0.76 & 0.83 & 0.59 & 0.58 & 0.53 & 0.83 & 0.53 \\
\hline
\end{tabular}

Table 9

Effect of valid inequalities on P\&K-M with partial coverage for the TR data set.

\begin{tabular}{|c|c|c|c|c|c|c|c|c|c|c|}
\hline$\alpha$ & $p$ & & No valid ineq & $(2.17)$ & $(3.15)$ & $(3.16)$ & (3.15), (3.16) & (2.17), (3.16) & (2.17), (3.15) & (2.17), (3.15), (3.16) \\
\hline \multirow[t]{2}{*}{0.8} & \multirow[t]{2}{*}{5} & Best bn at root node (\%) & 171.94 & 97.54 & 171.94 & 100.66 & 100.64 & 97.54 & 97.54 & 97.54 \\
\hline & & Gap (\%) & 66.80 & 2.54 & 68.40 & 11.20 & 2.85 & 2.48 & 2.22 & 2.22 \\
\hline \multirow[t]{2}{*}{0.8} & \multirow[t]{2}{*}{10} & Best bn at root node (\%) & 172.06 & 97.57 & 172.06 & 100.66 & 100.64 & 97.57 & 97.57 & 97.57 \\
\hline & & Gap (\%) & 57.80 & 0.60 & 56.20 & 0.46 & 1.61 & 0.60 & 0.48 & 0.49 \\
\hline \multirow[t]{2}{*}{0.9} & \multirow[t]{2}{*}{5} & Best bn at root node (\%) & 170.57 & 96.30 & 170.57 & 99.40 & 99.37 & 96.30 & 96.30 & 96.30 \\
\hline & & Gap (\%) & 69.80 & 2.35 & 69.00 & 1.49 & 7.85 & 2.27 & 1.59 & 1.59 \\
\hline \multirow[t]{2}{*}{0.9} & \multirow[t]{2}{*}{10} & Best bn at root node (\%) & 170.68 & 96.31 & 170.68 & 99.40 & 99.37 & 96.31 & 96.31 & 96.31 \\
\hline & & Gap (\%) & 52.00 & 0.26 & 57.30 & 0.23 & 0.30 & 0.25 & 0.25 & 0.26 \\
\hline
\end{tabular}

analysis. None of the eight instances is solved to optimality with the formulation in [18] within one hour, whereas with the basic formulation, the optimal solution is found for one instance. We also report the percentage of the best upper bound at the root node for both coverage types for all instances, and the value with P\&K-S is smaller than with the other two formulations (Appendix Table A1). We also note that for all instances, the percentages with the formulation in [18] are always more than $100 \%$, which is the objective value when all $O-D$ pairs are covered.

Similar analyses and conclusions are also valid for the partial coverage case for the $\mathrm{CAB}$ data set (Table 5). Although the solution times of the proposed formulation increase, they are still much better than the existing ones. Optimality is proved within three minutes with the proposed formulation (Appendix Table A1). 
We also compare the formulations with the TR data set for both coverage types and the results are presented in Table 6. The TR data set is relatively larger than the CAB data set, therefore we set a two-hour time limit. Since the existing formulations have $O\left(n^{4}\right)$ variables, we ran out of memory for both coverage types and could not obtain even the initial solutions. Therefore, we show only the results of the proposed formulation.

For binary coverage, all the instances are solved to optimality with P\&K-S within three minutes. For partial coverage, the solution times increase, but only in two instances ( $\alpha=0.8, p=5$ and $\alpha=0.9, p=5)$ is optimality not proved within two hours. For these instances, we report the $g a p(\%)$ that is reported by the solver at the end of the time limit. Also, independent from the coverage type, for each $\alpha$, the solution time decreases with the increment of $p$. Throughout the paper, the best integer values are written in parentheses when they are not optimally verified with any of the formulations.

We also conclude from Table A1 in the Appendix and Table 6 that for each $p$, the increment in $\alpha$ results in a decrement in optimal coverage for both coverage types. We also find that smaller $\alpha$ values generally give better coverage. For the TR data set, this result is consistent because the lower and upper time limits for each zone are the same, so as $\alpha$ increases, traversing the link between any two hubs also increases, and hence any $O-D$ pair turns out less likely to be covered than it is with smaller $\alpha$. However, for the CAB data set, this pattern does not always hold due to having different limits for different $\alpha$ and $p$ values. If we check the two instances $\alpha=0.2, p=2$ and $\alpha=0.4, p=2$, the maximum coverage is higher for the second one since the former has $\beta=2136$ and the latter has $\beta=2401$.

Last, we analyze the effect of allowing partial coverage; the complete results of P\&K-S with the CAB data set for all $\alpha$ values are presented in the Appendix Table A1. As well as optimal values and CPU times, we also provide hub locations. For the CAB data set, first we again emphasize that allowing partial coverage increases $\mathrm{CPU}$ time by about $50 \mathrm{~s}$.

When we compare the percentages of maximum coverage, as expected, the values for partial coverage are higher than the values for binary coverage, and on average, the increment is $3.22 \%$. One of the reasons for this increment is due to allowing partial coverage; even if all hub locations are the same for both coverage types, some of the $O-D$ pairs are partially covered, whereas they are not covered at all with binary coverage. For example, for the instance $\alpha=0.2, p=4$, the $O-D$ pairs given in Table 7 are the ones that are not covered with binary coverage $\left(Z_{i j}=Z_{j i}=0\right)$ and are partially covered when partial coverage is allowed. Thus, the increment in the objective function value is only due to these partially covered pairs. Recall that $Z_{i j}$ is the fraction of flow between $O-D$ pairs that is covered.

The other reason for this increment in optimum value is due to the change in hub locations and the change in the allocation of nonhub nodes. From Table A1 in the Appendix it can be observed that in half the instances, the hub locations change. For example, when partial coverage is allowed, for the instance with $\alpha=0.6$, $p=4$, three of the hub locations change (Fig. 3). With binary coverage, node 14 is selected as a hub, but with partial coverage node 13 is selected as a hub instead. This change is remarkable since the distance $\left(d_{13,14}\right)$ between nodes 13 and 14 is almost half the coverage distance and affects the objective value significantly. However, in some instances, the changes are not so important. For example, for the instance with $\alpha=0.4, p=5$, all the optimal hubs for partial coverage are the same as for binary coverage except for one; when partial coverage is allowed, the hub located at node 18 moves to the nearest node, 17 (Fig. 4).

From the Appendix, Table A1 it can be inferred that hub locations become more distant from each other when partial coverage is introduced. For the instance with $\alpha=0.6, p=4$, node 12 is selected as a hub for both coverage types; for binary coverage, nodes 11, 14 and 20 are chosen, and for partial coverage, nodes 13 ,
18 and 22 are chosen (Fig. 3). Spatially, the latter hubs are more distant from each other than the former ones. This result is similar to the other instances where the hub locations are different for both coverage types. Becoming more distant with partial coverage seems consistent with this type of coverage. Since longer distances can be covered by moving the hubs farther apart, more demands can be covered (but with poorer value). However, we remark here that the differences of the hub locations with binary and partial coverage for the $C A B$ data set are mainly due to having dispersed locations of nodes. So, the hub locations may not be so far away from each other with more dense locations of the nodes.

Note that hub locations generally move to larger cities such as New York (17) or San Francisco (22) when partial coverage is introduced. For the instance with $\alpha=0.6, p=3$, this is evident; instead of nodes 5 and 11, nodes 13 and 17 are selected as hubs. This case can also be seen for node 22. For some instances, for example $\alpha=0.6, p=5$, even if node 12 (the nearest node to 22) is a hub for binary coverage, 22 is also selected as a hub for partial coverage due to its size. And even if nodes 4 and 17 are hubs for binary coverage, the flows between node 12 and nodes $3,6,9,17$, 18,20 and 25 (more than one-third of its total flow) are not covered. Therefore, at partial coverage, node 22 is also selected. We also calculated the objective values for partial coverage using the optimal hub locations for binary coverage and found that the difference between the optimal objective value of partial coverage varies between $0.03 \%$ and $5 \%$. Therefore, we can conclude that with these parameter settings, the optimal locations of hubs with binary coverage also yield "good" solutions for partial coverage.

Next, we compare the solutions with the TR data set for the results for both coverage types in Table 6. The effect of partial coverage on the objective value and solution time is more evident for the TR data set. We note that the effect of partial coverage strongly depends on the parameters in binary and partial coverages and the effect of using partial coverage may increase or decrease depending on the functions used at $a_{i j}^{k m}$ and $b_{i j}^{k m}$. With the parameter settings given in Section 5.1, the difference between the solution times of both coverage types is higher than the difference with the $\mathrm{CAB}$ data set. Although the problem is solved within three minutes for binary coverage in all instances, for partial coverage there exist two instances whose optimality is not verified within the two-hour time limit. The importance of partial coverage can be observed easily from the results with the TR data set; on average a $15.06 \%$ improvement is obtained when partial coverage is allowed. Since the hub locations are the same for the instances where we obtained the optimal solutions for both coverage types, we do not present them here.

\section{Computational results for MApHMCP}

In this section, we first test the combination of valid inequalities for the proposed formulation. For MApHMCP, we compare results of the new formulation with the formulation in [6]. As explained in Section 3, the formulation in [31] works significantly better than the others since it has $\mathrm{O}\left(n^{2}\right)$ decision variables and constraints. However, since the formulation does not work with partial coverage, we did not perform any computational analyses using that model. We again argue the effect of partial coverage to the solutions and we compare the results of the proposed formulation with binary and partial coverage. Lastly, we discuss and compare the solutions of two problems in terms of cost and coverage that one of them has service oriented objective whereas other one has economic objective.

Similar to P\&K-S, the proposed formulation for MApHMCP can be improved for a symmetric distance matrix. Similarly, the objective function can again be changed to $\left(2.14^{\prime}\right)$ and $\forall i, j \in N$ : $i \leq j$ can be added to constraints (3.7)-(3.12) and to valid inequalities (2.17), (3.15) and (3.16). Additionally, from P\&K-M we can 
remove one set of binary variables for the route allocations since $X_{i j k}=Y_{j i k} \forall i, j \in N, k \in H$ for the symmetric distance matrix; thus, we remove $Y_{i j m}$ from the formulation. Therefore, the related constraints ((3.8), (3.10), (3.12) and (3.14)) are also removed. This change reduces the number of constraints by almost half. After removing $Y_{i j m}$, constraint (3.7) is substituted with

$Z_{i j} \leq \sum_{k \in H} a_{i j}^{k m} X_{i j k}+\lambda_{i j}\left(1-X_{j i m}\right) \quad \forall m \in H, \forall i, j \in N: i \leq j$

\subsection{Performance analysis of the proposed valid inequalities}

First, we report and compare the effect of the valid inequalities explained in Section 3 for P\&K-M. The preliminary analyses are given for binary and partial coverages with the TR data set for the combination of parameters of $\alpha=0.8,0.9$ and $p=5,10$.

Table 10

MApHMCP solutions with binary coverage for the CAB data set.

\begin{tabular}{|c|c|c|c|c|c|c|c|}
\hline \multirow[t]{2}{*}{$\alpha$} & \multirow[t]{2}{*}{$p$} & \multicolumn{3}{|l|}{ P\&K-M } & \multicolumn{3}{|l|}{ Campbell [6] } \\
\hline & & $\begin{array}{l}\text { Coverage } \\
(\%) / \text { best int } \\
(\%)\end{array}$ & $\begin{array}{l}\text { CPU } \\
\text { (sec)/gap } \\
(\%)\end{array}$ & $\begin{array}{l}\text { bb at } \\
\text { root } \\
(\%)\end{array}$ & $\begin{array}{l}\text { Coverage } \\
(\%) / \text { best int } \\
(\%)\end{array}$ & $\begin{array}{l}\text { CPU (sec)/ } \\
\text { gap (\%) }\end{array}$ & $\begin{array}{l}\text { bb at } \\
\text { root } \\
(\%)\end{array}$ \\
\hline \multirow[t]{4}{*}{0.2} & 2 & 93.10 & 95.85 & 99.95 & 86.873 & $>1 \mathrm{~h} / 15.10$ & 100 \\
\hline & 3 & 96.58 & 266.65 & 100 & 96.58 & $>1 \mathrm{~h} / 3.54$ & 100 \\
\hline & 4 & 95.71 & 1275 & 99.98 & 95.711 & $>1 \mathrm{~h} / 4.48$ & 100 \\
\hline & 5 & (92.70) & $>1 \mathrm{~h} / 1.5$ & 99.94 & (92.70) & $>1 \mathrm{~h} / 7.58$ & 100 \\
\hline \multirow[t]{4}{*}{0.4} & 2 & 96.04 & 85.75 & 99.89 & 94.46 & $>1 \mathrm{~h} / 5.86$ & 100 \\
\hline & 3 & 96.24 & 112.66 & 99.92 & 95.57 & $>1 \mathrm{~h} / 4.28$ & 100 \\
\hline & 4 & 95.01 & 395.94 & 99.92 & 95.01 & 1961.37 & 99.99 \\
\hline & 5 & 91.84 & 453.14 & 99.74 & 91.84 & 1346.49 & 99.10 \\
\hline \multirow[t]{4}{*}{0.6} & 2 & 93.96 & 69.67 & 99.76 & 93.96 & 1116.52 & 99.84 \\
\hline & 3 & 93.17 & 100.58 & 99.79 & 93.17 & 1044.94 & 99.33 \\
\hline & 4 & 93.63 & 101.91 & 99.77 & 93.64 & 724.6 & 98.41 \\
\hline & 5 & 90.19 & 139.82 & 96.50 & 90.19 & 767.52 & 94.26 \\
\hline \multirow[t]{4}{*}{0.8} & 2 & 89.92 & 103.71 & 96.88 & 89.92 & 1732.31 & 94.98 \\
\hline & 3 & 90.08 & 64.57 & 92.62 & 90.08 & 821.92 & 91.91 \\
\hline & 4 & 89.61 & 141.5 & 91.80 & 89.61 & 612.8 & 91.14 \\
\hline & 5 & 89.05 & 86.95 & 90.79 & 89.05 & 654.03 & 90.09 \\
\hline
\end{tabular}

The effect of valid inequality (2.17) is easily observed from Table 9. If the inequality is not added, there is a possibility of having the best bound at the root node higher than 100\% (columns 4 and 6-8). Our preliminary analyses show that for each instances in Tables 8 and 9, the lowest gaps are attained in columns 9 and 11; the differences between the column gaps are always lower than $1 \%$. For those columns, in order to have better selection criteria, we also consider the solutions after two hours. After that time, the gaps in column 9 (which uses the same valid inequality combinations for P\&K-S) gives slightly better results. Thus, we decide to use valid inequalities (2.17) and (3.16) in the computational experiments of P\&K-M.

\subsection{Computational experiments for MApHMCP}

For MApHMCP, we again report coverage(\%), bb at root(\%) and CPU if the solution is obtained within the time limit, and best int(\%) and $g a p(\%)$ if it is not solved to optimality within time limit. Observe that for binary coverage, for the $\mathrm{CAB}$ data set the proposed formulation is better than the formulation in [6] since the aim of that paper was to define and formulate the problem. In only one instance is the optimality not verified within one hour, thus resulting in a $1.5 \%$ gap at the end of one hour. For the same instance, the gap using the formulation in Campbell [6] is $7.58 \%$. For the remaining instances, the P\&K-M solution times are always shorter than the solution times of the formulation in [6] (Table 10).

The results of both formulations for partial coverage with the CAB data set are presented in Table 11. If we compare the CPU times for solved instances and the gaps for the others, in nine instances the proposed formulation results in a better solution. In terms of the solution quality, the proposed formulation cannot prove the optimality of seven instances out of 16 within the time limit, and the average gap of these instances is $1.42 \%$ at the end of one hour. On the other hand, the formulation in Campbell [6] cannot prove the optimality for five instances, and the average gap of those instances is $4.75 \%$. We also note that for MApHMCP the best upper bound value with the existing formulation provides slightly better results than the proposed one.

The results of the binary and partial coverages with the TR data sets are presented in Table 12. We again set a two-hour time limit. Similar to the single allocation version of the problem, Campbell

Table 11

MApHMCP solutions with partial coverage for the CAB data set.

\begin{tabular}{|c|c|c|c|c|c|c|c|}
\hline \multirow[t]{2}{*}{$\alpha$} & \multirow[t]{2}{*}{$p$} & \multicolumn{3}{|l|}{ P\&K-M } & \multicolumn{3}{|l|}{ Campbell [6] } \\
\hline & & Coverage (\%)/best int (\%) & CPU (sec)/gap (\%) & bb at root (\%) & Coverage (\%)/best int (\%) & CPU (sec)/gap (\%) & bb at root $(\%)$ \\
\hline \multirow[t]{4}{*}{0.2} & 2 & 97.01 & 520.35 & 100 & 93.36 & $>1 \mathrm{~h} / 7.11$ & 100 \\
\hline & 3 & 98.26 & 1962.78 & 100 & 98.24 & $>1 \mathrm{~h} / 1.79$ & 100 \\
\hline & 4 & $(97.14)$ & $>1 \mathrm{~h} / 0.78$ & 100 & $(92.67)$ & $>1 \mathrm{~h} / 7.90$ & 100 \\
\hline & 5 & $(94.91)$ & $>1 \mathrm{~h} / 4.40$ & 99.99 & $(94.91)$ & $>1 \mathrm{~h} / 4.60$ & 100 \\
\hline \multirow[t]{4}{*}{0.4} & 2 & 97.70 & 422.41 & 99.99 & 97.70 & $>1 \mathrm{~h} / 2.35$ & 100 \\
\hline & 3 & 97.23 & 2885.75 & 99.99 & 97.23 & 3461.27 & 100 \\
\hline & 4 & 96.90 & $>1 \mathrm{~h} / 0.67$ & 99.98 & 96.90 & 2179.51 & 100 \\
\hline & 5 & 95.12 & $>1 \mathrm{~h} / 1.75$ & 99.89 & 95.12 & 1819.72 & 99.66 \\
\hline \multirow[t]{4}{*}{0.6} & 2 & 96.2 & 547.99 & 99.95 & 96.2 & 1069.59 & 99.95 \\
\hline & 3 & 95.74 & 3369.32 & 99.95 & 95.74 & 1358.43 & 99.82 \\
\hline & 4 & 96.12 & $>1 \mathrm{~h} / 0.61$ & 99.93 & 96.12 & 1536.86 & 99.45 \\
\hline & 5 & 94.61 & $>1 \mathrm{~h} / 1.33$ & 98.96 & 94.61 & 1145.15 & 97.66 \\
\hline \multirow[t]{4}{*}{0.8} & 2 & 94.16 & 618.99 & 99.20 & 94.16 & 1064.1 & 98.26 \\
\hline & 3 & 93.66 & 790.15 & 97.39 & 93.66 & 969.32 & 96.62 \\
\hline & 4 & 93.58 & 2587.47 & 96.96 & 93.58 & 985.04 & 95.46 \\
\hline & 5 & 91.51 & $>1 \mathrm{~h} / 0.43$ & 93.91 & 91.51 & 878.69 & 92.83 \\
\hline
\end{tabular}


[6] has $O\left(n^{4}\right)$ variables and constraints, so with the TR data set for both coverage types, the formulation cannot generate an initial solution due to the memory requirements. Therefore, we only present the results of the proposed formulation.

For binary coverage, we obtain five optimal solutions out of eight within the two-hour time limit. The gaps for the remaining instances vary between $0.29 \%$ and $2.24 \%$. For partial coverage, we obtain one fewer optimal solution, and the gap for that instance $(\alpha=0.9, p=10)$ is $0.07 \%$ at the end of the time limit. The gaps for partial coverage are slightly smaller than the gaps for binary coverage, with the maximum gap for partial coverage $2.17 \%$. For the TR data set, we observe from the results that the gaps decrease with the increment of $p$ for each $\alpha$ for both coverage types, and finally, for $p=15$ and 20, the gaps become zero. Similarly, the solution times for each $\alpha$ for the instances with $p=15$ and 20 decrease with the increment of $p$; the CPUs of the instances with $p=15$ are smaller than the CPUs of the instances with $p=20$ for each $\alpha$.

A similar observation for the relationship between $\alpha$ and optimal value holds for multiple allocation for both data sets and both coverage types (Table 12 and Appendix, Table A2). Generally, an increment in $\alpha$ yields a decrement in the optimal solution, with

Table 12

MApHMCP solutions with binary and partial coverage types for the TR data set.

\begin{tabular}{|c|c|c|c|c|c|}
\hline \multirow[t]{2}{*}{$\alpha$} & \multirow[t]{2}{*}{$p$} & \multicolumn{2}{|l|}{ Binary } & \multicolumn{2}{|l|}{ Partial } \\
\hline & & $\begin{array}{l}\text { Coverage (\%)/best } \\
\text { int (\%) }\end{array}$ & $\begin{array}{l}\text { CPU (sec)/ } \\
\text { gap (\%) }\end{array}$ & $\begin{array}{l}\text { Coverage (\%)/best } \\
\text { int }(\%)\end{array}$ & $\begin{array}{l}\text { CPU (sec)/ } \\
\text { gap (\%) }\end{array}$ \\
\hline \multirow[t]{4}{*}{0.8} & 5 & (84.59) & $>1 \mathrm{~h} / 2.24$ & $(95.51)$ & $>1 \mathrm{~h} / 1.88$ \\
\hline & 10 & $(88.63)$ & $>1 \mathrm{~h} / 0.29$ & (97.15) & $>1 \mathrm{~h} / 0.29$ \\
\hline & 15 & 89.97 & 1241.52 & 97.49 & 5581.7 \\
\hline & 20 & 90.33 & 32.17 & 97.58 & 81.34 \\
\hline \multirow[t]{4}{*}{0.9} & 5 & $(82.63)$ & $>1 \mathrm{~h} / 1.21$ & $(94.10)$ & $>1 \mathrm{~h} / 2.17$ \\
\hline & 10 & 84.47 & 2097.88 & $(96.12)$ & $>1 \mathrm{~h} / 0.07$ \\
\hline & 15 & 85.01 & 239.55 & 96.25 & 3463.75 \\
\hline & 20 & 85.25 & 144.85 & 96.31 & 111.53 \\
\hline
\end{tabular}

the only exception occurring with the same instances as single allocation ( $\alpha=0.2, p=2$ and $\alpha=0.4, p=2$ ). Again, the optimal value for $\alpha=0.4, p=2$ is higher than the optimal value for $\alpha=0.2, p=2$.

Next, we analyze the effect of allowing partial coverage to MApHMCP, with the results for P\&K-M for the CAB data set shown in the Appendix, Table A2. First, again observe that, as expected, the objective values increase for each instance, and on average, the increment is $2.92 \%$ when partial coverage is allowed. Note that the average increment may change (either increase or decrease) since there is a chance of having a higher coverage value than the given best integer solutions for the three instances. The increment in solution time is evident in the Appendix, Table A2. The increment in solution time also leads to an increment in the number of unsolved instances for partial coverage.

We also compare the difference in hub locations for both coverage types. Since optimality is not proved for partial coverage for the two instances ( $\alpha=0.2, p=4$ and $\alpha=0.2, p=5$ ), we do not consider them. Therefore, in 11 instances out of 14 , the hub locations change when partial coverage is allowed. From Table A2 it can be inferred that some changes in hub locations are not significant; for five instances, only one of the hubs with partial coverage is different than the hubs with binary coverage. Generally, the new hub with partial coverage is the nearest node to the hub with binary coverage. For example, for the instance with $\alpha=0.2, p=3$, all hubs are same except node 12 ; in the partial coverage case, node 22 (the nearest node to 12 ) is selected instead.

Similar conclusions as explained in Section 5 for SApHMCP also hold for the multiple allocation version of the problem. When partial coverage is allowed, hubs become more distant from each other because the distances are greater than in binary coverage. For example, for the instance with $\alpha=0.8, p=4$, for binary coverage the longest distance is between hubs 1 and 22; for partial coverage it is between hubs 17 and 22 . We again note here that the differences of the hub locations with binary and partial coverage for the $C A B$ data set are mainly due to the having dispersed locations of nodes. Moreover, similar to the discussion regarding SApHMCP, hubs are more likely to be located in (or near) larger cities. This deduction can be easily verified with the instances for $\alpha=0.4, p=4$ and $\alpha=0.8, p=5$, where

Table 13

Comparison of $p$-hub median and $p$-hub maximal covering problems.

(a) Results of the single allocation $p$-hub median and SApHMCP with binary coverage for the CAB data set

\begin{tabular}{|c|c|c|c|c|c|c|c|}
\hline \multirow[t]{2}{*}{$\alpha$} & \multirow[t]{2}{*}{$p$} & \multicolumn{3}{|l|}{ p-hub median } & \multicolumn{3}{|c|}{ p-hub maximal covering } \\
\hline & & Optimal hubs & Min cost $\left(\times 10^{3}\right)$ & Coverage value of solution (\%) & Optimal hubs & Max coverage (\%) & Cost value of solution $\left(\times 10^{3}\right)$ \\
\hline \multirow[t]{4}{*}{0.8} & 2 & 12,20 & 1294.08 & 80.59 & 8,21 & 87.79 & 1477.23 \\
\hline & 3 & $2,4,12$ & 1158.83 & 82.36 & $5,11,12$ & 87.35 & 1308.50 \\
\hline & 4 & $1,4,12,18$ & 1087.66 & 82.73 & $5,11,19,22$ & 87.30 & 1350.87 \\
\hline & 5 & $1,4,7,12,18$ & 1034.1 & 82.66 & $1,9,11,12,22$ & 86.23 & 1313.34 \\
\hline
\end{tabular}

(b) Results of the $p$-hub median problem and $p$-hub maximal covering problem with different coverage and cost constraints

\begin{tabular}{lllll}
\hline$p$-hub median & & & $p$-hub maximal covering \\
Coverage $\geq$ & Cost value with coverage constraint $\left(\times 10^{3}\right)$ & & cost $\leq\left(\times 10^{3}\right)$ & coverage value with cost constraint $(\%)$ \\
\hline & 1342.31 & & 1087.66 & 82.73 \\
87.30 & 1298.19 & 1114.85 & 85.17 \\
86.43 & 1211.56 & 1142.04 & 85.42 \\
85.55 & 1095.26 & 1169.23 & 85.52 \\
84.68 & 1095.09 & 1196.43 & 85.52 \\
83.81 & 1095.09 & 1223.62 & 85.74 \\
82.94 & 1087.66 & 1250.81 & 86.06 \\
82.06 & & 1278.00 & 86.38 \\
& & 1305.19 & 87.04 \\
\end{tabular}


node 23 is selected as the hub that can be considered the farthest from any other node, but for none of the solutions with binary coverage is node 23 selected as a hub. The situation is also evident for the instance with $\alpha=0.8, p=4$ : for partial coverage, nodes 12 and 17 are again chosen as hubs instead of nodes 1 and 19 , which were selected as hubs for binary coverage.

As a direct result of the changes in hub locations, the assignments (and the routes for each $O-D$ pair) are also affected. Therefore, both increments and decrements occur in the value of the pair coverage and we investigate one of the solutions of an instance with $\alpha=0.4, p=4$. We compare the fraction of flow that is routed from/to node 23 and for binary coverage only the three pairs $\{12,23\},\{19,23\}$ and $\{22,23\}$ are covered. But for partial coverage, each pair such that $(i, 23)$ or $(23, i)$ is fully covered for $i=1 \ldots 25$ except node 14 . Even though node 14 is the farthest node from 23 , the $O-D$ pair is partially covered $\left(Z_{13,24}=\right.$ $\left.Z_{24,13}=0.25\right)$. There are also decrements in coverage values; $O-D$ pairs $\{8,14\},\{14,19\},\{14,22\},\{15,19\}$ and $\{15,22\}$ are covered for the binary coverage case, but are not covered at all with located hubs for the partial coverage case.

\subsection{Discussion about cost and coverage of the p-hub maximal covering problem}

As noted in the first section, the $p$-hub maximal covering problem only has a service level objective and does not consider any economic aspects in its pure form. However, because problems that do have economic objectives, such as the $p$-hub median problem, can lead to unsatisfactory results in terms of coverage, to increase the service level one may focus on covering objectives. We decided to compare the service networks of these two objectives, thus we compared the results of the $p$-hub median and the $p$-hub maximal covering problems in terms of hub locations, cost value and coverage percentages for $\alpha=0.8$ and $p=2-5$ for the CAB data set. For this purpose, we used single allocation and binary coverage with the same parameters given in Section 5.2. After obtaining the hub locations of the $p$-hub median problem, we calculated the coverage value of the resulting network, and similarly, after obtaining the hub locations of SAPHMCP, we calculated the associated cost value and they are presented at columns 5 and 8 respectively (Table 13a). For example, for $p=4$, the optimal hub locations for the $p$-hub median problem are $1,4,12$ and 18 with an objective value of $1087.66\left(\times 10^{3}\right)$. The associated coverage percentage of the network is $82.73 \%$. However, when the same instance is solved with the $p$-hub maximal covering problem, the optimal hub locations are $5,11,19$ and 22 giving a maximum coverage percentage $87.30 \%$. For this solution, the cost is calculated and it is equal to $1350.87\left(\times 10^{3}\right)$.

From the table, the trade-off between cost and coverage is easily observed. As expected, the $p$-hub maximal covering problem leads to better coverage and more cost than the $p$-hub median problem; and the $p$-hub median version provides the best possible cost with less coverage. If we allow more cost, a $6.21 \%$ improvement can be achieved with the $p$-hub maximal covering problem. Note that, optimal hub locations for the $p$-hub median problem tend to be at large cities such as 4 (Chicago) or 12 (Los Angeles) to minimize the total cost. However, optimal hub locations for the $p$-hub maximal covering problem are not need to be at large cities and can be located at more centralized cities.

We remark here that, this analysis still uses a single objective; either minimizing the cost or maximizing the coverage. We extend this analysis by including other objective as a constraint with the best attained values. That is, we solve the $p$-hub maximal covering problem with a cost constraint and $p$-hub median problem by adding a coverage constraint. For example, for the same instance $(p=4)$ we solve the $p$-hub maximal covering problem with a cost of less than or equal to $1087.66\left(\times 10^{3}\right)$ and $p$-hub median problem with a minimum coverage percentage of $87.30 \%$. The solutions of the eight instances in Table 13a are the same with the solutions when the constraints are added except $p=4$ and 5 for the $p$-hub median problem. For $p=4$, the minimum cost of the solution that satisfies $87.30 \%$ coverage is $1342.31\left(\times 10^{3}\right)$ and for $p=5$, the minimum cost of the solution that satisfies $86.23 \%$ coverage is $1241.01\left(\times 10^{3}\right)$. From this analysis, we conclude that adding these constraints to the problems can yield better solutions rather than the calculated value of the optimal solution.

Since we observe differences in the solution of the problems with and without the constraints, we further analyze the effect of adding cost and coverage constraints. Therefore, beside using the best attained values, the problems are solved with satisfying different coverage percentages and cost values. From Table 13b, generally, when lower coverage requirement is allowed at the $p$ hub median problem, the minimum cost decreases and similarly, the maximum coverage percentage increases with the increment of the cost value. However, from the results, it can be observed that, even if we increase (decrease) the right-hand side of the constraints, the maximum coverage percentage (minimum cost value) does not change. For instance, the $p$-hub median problem provides the same results that satisfy two different coverage percentages (83.81\% and $82.94 \%$ ).

As stated, in the $p$-hub maximal covering problem, the cost is underestimated and the optimal solution is obtained because there is no cost to establish links between nonhub nodes and hubs or between hubs. With this assumption, using single allocation seems useless because multiple allocation gives better coverage. We calculated the number of spoke links (links between nonhub nodes and hubs) for both allocations and both coverage types (Appendix Table A3). For the single allocation, number of spoke links is the same ( $n-p$ links) regardless of the coverage type. For the multiple allocation we calculated them for both coverage types because they can increase or decrease depending on hub locations. For multiple allocation, even assuming there is no cost to the links, any nonhub node can be assigned to all the hubs even if there is no flow between them or even if the time between them is larger than the threshold value. Moreover, we also observed that with the parameters that we used in the computational analysis, many alternative solutions exist due to assigning almost all nonhub nodes to every hub, although some are them are not necessary. Therefore, we report the number of spoke links that are needed and used (Appendix, Table A3).

For each instance, as expected, the number of spoke links with single allocation is fewer than the number of spoke links with multiple allocation because the latter is a relaxation of the former. So, if we want to calculate the cost of establishing these links, with the assumption that each of them has equal weight, the cost of the links with multiple allocation will be higher than the cost of the links with single allocation. Similar to the previous discussion, if costs exists, there is a trade-off between cost and coverage with the single and multiple allocations of the $p$-hub maximal covering problem: MApHMCP gives better coverage but with more spoke links than SApHMCP. Hence, a carrier that wants "good" coverage but still wants to consider the cost of establishing the links might choose SApHMCP rather than MApHMCP.

From the same table, we can also discuss the effect of having partial coverage on the number of spoke links for MApHMCP. Generally, the number of spoke links increases with the use of partial coverage. For seven out of 14 instances, the number of spoke links increases when partial coverage is used instead of binary coverage. (We do not count the two instances $\alpha=0.2 p=4,5$ where optimality is not proved.) However, we cannot say that with partial coverage the number always increases, because for five instances it remains the same and for three instances it decreases. So, the number of spoke links not only depends on the type of coverage, it also depends on hub locations. We can only discuss that even if hub locations are the same for both coverage types, there is no guarantee of an increment; for the instance $\alpha=0.6, p=4$, the number of spoke links is the same for both coverage types. 


\section{Conclusion}

In this paper, we studied SApHMCP and MApHMCP and observed that in the hub location literature, only binary coverage is taken into account. We extended the definition of coverage and introduced partial coverage to the hub location literature. We developed efficient mixed-integer programming formulations for both allocation types that can also be applied for partial coverage. In this paper, we also proved that both problems are NP-Hard. Further, we showed that the assignment version of SAPHMCP is NP-Hard.

Extensive computational study was carried out with the $\mathrm{CAB}$ and TR data sets. Based on the computational results, we conclude that both formulations generally perform better than the existing formulations. Thanks to the decrement in the number of variables and constraints, we obtain optimal (or near-optimal) solutions for a larger data set. We also discuss the impact of introducing partial coverage and present the results for both coverage types. As expected, the coverage percentages increase when partial coverage is allowed and for the TR data set, for SApHMCP, on the average $15.06 \%$ increment is calculated. We also compared the hub locations and observed that for the CAB data set, in the partial coverage case hubs are more likely located at or towards larger cities and they are more distant from each other. Lastly, we discuss briefly the $p$-hub median and $p$-hub maximal covering problem and we obtain that if having greater costs is allowed, higher service levels are obtained.

\section{Acknowledgements}

We sincerely thank the two anonymous referees for contributing to the improvement of our paper. The corresponding author gratefully acknowledges support from the Turkish Academy of Sciences.

Table A1

P\&K-S solutions for binary and partial coverage types for the CAB data set.

\begin{tabular}{|c|c|c|c|c|c|c|c|c|c|}
\hline \multirow[t]{2}{*}{$\alpha$} & \multirow[t]{2}{*}{$p$} & \multicolumn{4}{|c|}{ Binary coverage } & \multicolumn{4}{|c|}{ Partial coverage } \\
\hline & & Coverage (\%) & $\mathrm{CPU}(\mathrm{sec})$ & $\mathrm{bb}$ at root (\%) & Hub locations & Coverage (\%) & $\mathrm{CPU}(\mathrm{sec})$ & bb at root (\%) & Hub locations \\
\hline \multirow[t]{4}{*}{0.2} & 2 & 92.66 & 16.83 & 99.33 & 5,12 & 96.19 & 13.96 & 99.97 & 5,22 \\
\hline & 3 & 95.97 & 11.24 & 99.91 & $2,12,13$ & 97.76 & 15.80 & 100 & $2,13,22$ \\
\hline & 4 & 95.67 & 10.75 & 99.43 & $1,2,11,12$ & 97.07 & 21.14 & 99.99 & $1,2,11,12$ \\
\hline & 5 & 92.38 & 9.11 & 97.99 & $4,7,12,18,24$ & 94.68 & 67.87 & 99.92 & $4,7,12,18,24$ \\
\hline \multirow[t]{4}{*}{0.4} & 2 & 94.01 & 9.03 & 98.59 & 5,12 & 96.53 & 16.89 & 99.88 & 5,12 \\
\hline & 3 & 95.40 & 8.52 & 98.04 & $12,13,17$ & 96.72 & 23.91 & 99.90 & $12,13,17$ \\
\hline & 4 & 94.39 & 8.46 & 97.41 & $11,12,14,18$ & 96.49 & 29.85 & 99.85 & $11,12,14,18$ \\
\hline & 5 & 89.19 & 5.68 & 93.24 & $12,18,21,22,24$ & 93.40 & 149.97 & 99.43 & $12,17,21,22,24$ \\
\hline \multirow[t]{4}{*}{0.6} & 2 & 90.01 & 8.93 & 95.11 & 5,12 & 93.47 & 29.05 & 99.63 & 5,12 \\
\hline & 3 & 91.91 & 3.7 & 93.82 & $5,11,12$ & 94.02 & 38.76 & 99.65 & $12,13,17$ \\
\hline & 4 & 91.51 & 4.65 & 93.74 & $11,12,14,20$ & 94.62 & 43.84 & 99.58 & $12,13,18,22$ \\
\hline & 5 & 88.38 & 3.28 & 90.63 & $4,7,12,17,24$ & 93.00 & 131.1 & 98.46 & $11,12,18,22,24$ \\
\hline \multirow[t]{4}{*}{0.8} & 2 & 87.79 & 1.06 & 89.53 & 8,21 & 91.83 & 26.95 & 98.61 & 8,21 \\
\hline & 3 & 87.35 & 2.16 & 89.15 & $5,11,12$ & 90.86 & 50.27 & 96.87 & $5,11,12$ \\
\hline & 4 & 87.30 & 4.36 & 89.21 & $5,11,19,22$ & 90.87 & 116.57 & 96.51 & $8,12,17,21$ \\
\hline & 5 & 86.23 & 2.02 & 87.48 & $1,9,11,12,22$ & 89.29 & 132.42 & 93.51 & $6,8,11,12,24$ \\
\hline
\end{tabular}

Table A2

P\&K-M solutions for binary and partial coverage types for the CAB data set.

\begin{tabular}{|c|c|c|c|c|c|c|c|}
\hline \multirow[t]{2}{*}{$\alpha$} & \multirow[t]{2}{*}{$p$} & \multicolumn{3}{|c|}{ Binary coverage } & \multicolumn{3}{|l|}{ Partial coverage } \\
\hline & & Coverage (\%) & CPU (sec)/gap (\%) & Hub locations & Coverage (\%)/best int (\%) & CPU (sec)/gap (\%) & Hub locations \\
\hline \multirow[t]{4}{*}{0.2} & 2 & 93.10 & 95.85 & 5,12 & 97.01 & 520.35 & 5,22 \\
\hline & 3 & 96.58 & 266.65 & $2,12,13$ & 98.26 & 1962.78 & $2,13,22$ \\
\hline & 4 & 95.71 & 1275 & $1,11,12,25$ & $(97.14)$ & $>1 \mathrm{~h} / 0.78$ & $1,11,12,25$ \\
\hline & 5 & $(92.70)$ & $>1 \mathrm{~h} / 1.5$ & $4,7,12,18,24$ & (94.91) & $>1 \mathrm{~h} / 4.40$ & $4,7,12,18,24$ \\
\hline \multirow[t]{4}{*}{0.4} & 2 & 96.04 & 85.75 & 5,12 & 97.70 & 422.41 & 5,12 \\
\hline & 3 & 96.24 & 112.66 & $12,13,20$ & 97.23 & 2885.75 & $12,13,17$ \\
\hline & 4 & 95.01 & 395.94 & $11,12,14,18$ & 96.90 & $>1 \mathrm{~h} / 0.67$ & $12,13,18,23$ \\
\hline & 5 & 91.84 & 453.14 & $1,2,11,12,22$ & 95.12 & $>1 \mathrm{~h} / 1.75$ & $11,12,18,22,24$ \\
\hline \multirow[t]{4}{*}{0.6} & 2 & 93.96 & 69.67 & 5,12 & 96.20 & 547.99 & 5,12 \\
\hline & 3 & 93.17 & 100.58 & $5,8,12$ & 95.74 & 3369.32 & $11,12,25$ \\
\hline & 4 & 93.63 & 101.91 & $12,13,18,22$ & 96.12 & $>1 \mathrm{~h} / 0.61$ & $12,13,18,22$ \\
\hline & 5 & 90.19 & 139.82 & $4,12,16,17,22$ & 94.61 & $>1 \mathrm{~h} / 1.33$ & $11,12,17,22,24$ \\
\hline \multirow[t]{4}{*}{0.8} & 2 & 89.92 & 103.71 & 6,8 & 94.16 & 618.99 & 8,20 \\
\hline & 3 & 90.08 & 64.57 & $2,11,12$ & 93.66 & 790.15 & $11,12,20$ \\
\hline & 4 & 89.61 & 141.5 & $1,4,19,22$ & 93.58 & 2587.47 & $12,17,21,22$ \\
\hline & 5 & 89.05 & 86.95 & $1,4,8,12,22$ & 91.51 & $>1 \mathrm{~h} / 0.43$ & $12,21,22,23,25$ \\
\hline
\end{tabular}


Table A3

Number of spoke links in optimal solutions for the CAB data sets.

\begin{tabular}{|c|c|c|c|c|}
\hline \multirow[t]{2}{*}{$\alpha$} & \multirow[t]{2}{*}{$p$} & \multirow{2}{*}{$\begin{array}{l}\text { SA } \\
\text { Binary \& Partial }\end{array}$} & \multicolumn{2}{|l|}{ MA } \\
\hline & & & Binary & Partial \\
\hline \multirow[t]{4}{*}{0.2} & 2 & 23 & 24 & 24 \\
\hline & 3 & 22 & 23 & 23 \\
\hline & 4 & 21 & 22 & 22 \\
\hline & 5 & 20 & 22 & 24 \\
\hline \multirow[t]{4}{*}{0.4} & 2 & 23 & 26 & 26 \\
\hline & 3 & 22 & 24 & 23 \\
\hline & 4 & 21 & 22 & 25 \\
\hline & 5 & 20 & 25 & 29 \\
\hline \multirow[t]{4}{*}{0.6} & 2 & 23 & 28 & 30 \\
\hline & 3 & 22 & 31 & 32 \\
\hline & 4 & 21 & 27 & 27 \\
\hline & 5 & 20 & 27 & 29 \\
\hline \multirow[t]{4}{*}{0.8} & 2 & 23 & 34 & 36 \\
\hline & 3 & 22 & 31 & 30 \\
\hline & 4 & 21 & 33 & 28 \\
\hline & 5 & 20 & 30 & 31 \\
\hline
\end{tabular}

\section{Appendix A}

See Tables A1-A3.

\section{References}

[1] Adenso-Diaz B, Rodriguez F. A simple search heuristic for the MCLP: application to the location of ambulance bases in a rural region. Omega 1997; 25(2):181-7.

[2] Alumur SA, Kara BY. Network hub location problems: the state of the art. European Journal of Operational Research 2008;190(1):1-21.

[3] Berman O, Krass D. The generalized maximal covering location problem. Computers and Operations Research 2002;29(6):563-81.

[4] Berman O, Krass D, Drezner Z. The gradual covering decay location problem on a network. European Journal of Operational Research 2003;151(3):474-80.

[5] Calik H, Alumur SA, Kara BY, Karasan OE. A tabu-search based heuristic for the hub covering problem over incomplete hub networks. Computers and Operations Research 2009;36(12):3088-96.

[6] Campbell JF. Integer programming formulations of discrete hub location problems. European Journal of Operational Research 1994;72(2):387-405.

[7] Campbell JF. Modeling economies of scale in transportation hub networks. In: 2013 46th Hawaii International Conference on System Sciences (HICSS), IEEE; 2013. p. $1154-63$
[8] Campbell JF, O'Kelly ME. Twenty-five years of hub location research. Transportation Science 2012:46(2):153-69.

[9] Campbell JF, Ernst AT, Krishnamoorthy M. Hub arc location problems: Part Iintroduction and results. Management Science 2005;51(10):1540-55.

[10] Chen J. A hybrid heuristic for the uncapacitated single allocation hub location problem. Omega 2007;35(2):211-20.

[11] Church R, ReVelle C. The maximal covering location problem. Papers in Regional Science 1974;32(1):101-18.

[12] Church RL, Roberts KL. Generalized coverage models and public facility location. In: Papers of the regional science association, vol. 53. Springer; 1983. p. $117-35$.

[13] Costa AM, França PM, Lyra Filho C. Two-level network design with intermediate facilities: an application to electrical distribution systems. Omega 2011; 39(1):3-13.

[14] Ernst AT, Hamacher H, Jiang H, Krishnamoorthy M, Woeginger G. Uncapacitated single and multiple allocation p-hub center problems. Computers and Operations Research 2009;36(7):2230-41.

[15] Ernst AT, Krishnamoorthy M, Jiang H, Baatar D. Reformulations and computational results for the uncapacitated single allocation hub covering problem. Unpublished report; 2011.

[16] Farahani RZ, Asgari N, Heidari N, Hosseininia M, Goh M. Covering problems in facility location: a review. Computers and Industrial Engineering 2012; 62(1):368-407.

[17] Galvao RD, ReVelle C. A Lagrangean heuristic for the maximal covering location problem. European Journal of Operational Research 1996;88(1):114-23.

[18] Hwang YH, Lee YH. Uncapacitated single allocation p-hub maximal covering problem. Computers and Industrial Engineering 2012:63(2):382-9.

[19] Kara BY, Taner MR. Hub location problems: the location of interacting facilities In: Foundations of location analysis, New York: Springer; 2011. p. 273-88.

[20] Kara BY, Tansel BC. On the single-assignment p-hub center problem. European Journal of Operational Research 2000;125(3):648-55.

[21] Kara BY, Tansel BC. The single-assignment hub covering problem: models and linearizations. Journal of the Operational Research Society 2003;54(1):59-64.

[22] Karasakal O, Karasakal EK. A maximal covering location model in the presence of partial coverage. Computers and Operations Research 2004;31(9):1515-26.

[23] Ma H, Miao Z, Lim A, Rodrigues B. Crossdocking distribution networks with setup cost and time window constraint. Omega 2011;39(1):64-72.

[24] Megiddo N, Zemel E, Hakimi SL. The maximum coverage location problem. SIAM Journal on Algebraic Discrete Methods 1983;4(2):253-61.

[25] O'Kelly ME. The location of interacting hub facilities. Transportation Science 1986:20(2):92-106.

[26] O'Kelly ME. A quadratic integer program for the location of interacting hub facilities. European Journal of Operational Research 1987;32(3):393-404.

[27] O'Kelly ME, Bryan DL. Hub location with flow economies of scale. Transportation Research Part B: Methodological 1998;32(8):605-16.

[28] Qu B, Weng K. Path relinking approach for multiple allocation hub maxima covering problem. Computers and Mathematics with Applications 2009; 57(11):1890-4.

[29] Tan PZ, Kara BY. A hub covering model for cargo delivery systems. Networks 2007;49(1):28-39.

[30] Wagner B. Model formulations for hub covering problems. Journal of the Operational Research Society 2008;59(7):932-8.

[31] Weng K, Yang C, Ma Y. Two artificial intelligence heuristics in solving multiple allocation hub maximal covering problem. In: Intelligent computing, Springer 2006. p. 737-44.

[32] Yaman H, Karasan OE, Kara BY. Release time scheduling and hub location for next-day delivery. Operations Research 2012;60(4):906-17. 